\title{
The supergiant fast X-ray transients XTE J1739-302 and IGR J08408-4503 in quiescence with XMM-Newton
}

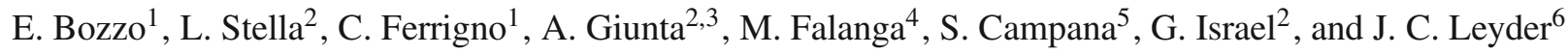 \\ ${ }^{1}$ ISDC - Science Data Centre for Astrophysics, University of Geneva, Chemin d'Écogia 16, 1290 Versoix, Switzerland \\ e-mail: enrico.bozzo@unige.ch \\ 2 INAF - Osservatorio Astronomico di Roma, via Frascati 33, 00044 Rome, Italy \\ Dipartimento di Fisica - Università di Roma Tor Vergata, via della Ricerca Scientifica 1, 00133 Rome, Italy \\ 4 International Space Science Institute (ISSI) Hallerstrasse 6, 3012 Bern, Switzerland \\ 5 INAF - Osservatorio Astronomico di Brera, via Emilio Bianchi 46, 23807 Merate (LC), Italy \\ ${ }^{6}$ Institut d'Astrophysique et de Géophysique de l'Université de Liège, 17 allée du 6 août, 4000 Liège, Belgium
}

Received 18 January 2010 / Accepted 9 April 2010

ABSTRACT

\begin{abstract}
Context. Supergiant fast X-ray transients are a subclass of high mass X-ray binaries that host a neutron star accreting mass from the wind of its OB supergiant companion. They are characterized by an extremely pronounced and rapid variability in X-rays, which still lacks an unambiguous interpretation. A number of deep pointed observations with XMM-Newton have been carried out to study the quiescent emission of these sources and gain insight into the mechanism that causes their X-ray variability.

Aims. We continued this study by using three XMM-Newton observations of the two supergiant fast X-ray transient prototypes XTE J1739-302 and IGR J08408-4503 in quiescence.

Methods. An in-depth timing and spectral analysis of these data have been carried out.

Results. We found that the quiescent emission of these sources is characterized by both complex timing and spectral variability, with multiple small flares occurring sporadically after periods of lower X-ray emission. Some evidence is found in the XMM-Newton spectra of a soft component below $\sim 2 \mathrm{keV}$, similar to that observed in the two supergiant fast X-ray transients AX J1845.0-0433 and IGR J16207-5129 and in many other high mass X-ray binaries.

Conclusions. We suggest some possible interpretations of the timing and spectral properties of the quiescent emission of XTE J1739302 and IGR J08408-4503 in the context of the different theoretical models proposed to interpret the behavior of the supergiant fast $\mathrm{X}$-ray transients.
\end{abstract}

Key words. X-rays: binaries - stars: individual: XTE J1739-302 - stars: individual: IGR J08408-4503 - stars: neutron - X-rays: stars

\section{Introduction}

Supergiant fast X-ray transients are a subclass of supergiant $\mathrm{X}$-ray binaries (SGXBs) that host a neutron star (NS) accreting from the wind of its OB supergiant companion (SFXT, Sguera et al. 2005). In contrast to the previously known supergiant X-ray binaries, SGXBs (i.e., the so-called "classical SGXBs", see e.g., White et al. 1995), SFXTs are characterized by a pronounced transient-like activity. These sources undergo few hours long (as opposed to weeks-months long) outbursts with peak X-ray luminosities of $\gtrsim 10^{36} \mathrm{erg} \mathrm{s}^{-1}$, and exhibit a large dynamic range in X-ray luminosity $\left(\gtrsim 10^{4}\right.$ between outburst and quiescence; Walter \& Zurita Heras 2007). The origin of this extreme variability is still debated, and different models have been developed to interpret it. One of these models involves a NS accreting matter from the extremely clumpy wind of its supergiant companion (in't Zand 2005; Walter \& Zurita Heras 2007; Negueruela et al. 2008). According to this interpretation, the sporadic capture and accretion of these clumps by the compact object can produce the observed fast X-ray flares. To reach the required $\mathrm{X}$-ray luminosity swing, very high density clumps are required (a factor $10^{4}-10^{5}$ denser than the homogeneous stellar wind, Walter \& Zurita Heras 2007). Numerical simulations of supergiant star winds indicate that these high density clumps might be produced by instabilities in the wind (Runacres \& Owocki 2002;
Oskinova et al. 2007, and references therein). Bozzo et al. (2008) proposed that the X-ray variability of the SFXT sources might be driven by centrifugal and/or magnetic "gating" mechanisms that can halt most of the accretion flow during quiescence, and only occasionally permit direct accretion onto the NS (see also Grebenev \& Sunyaev 2007). The properties of these gating mechanisms depend mainly on the NS magnetic field and spin period. At odds with the extremely clumpy wind model, in the gating scenario a transition from the regime in which the accretion is (mostly) inhibited to that in which virtually all the captured wind material accretes onto the NS requires comparatively small variations in the stellar wind velocity and/or density, and can easily give rise to a very large dynamic range in X-ray luminosity. Yet another model was proposed for IGR J11215-5952, so far the only SFXT to display regular periodic outbursts, which envisages that the outbursts take place when the NS in its orbit crosses a high density equatorial region in the supergiant's wind (Sidoli et al. 2007).

Except for their peculiar X-ray variability, SFXT sources share many properties with the previously known SGXBs. Measured orbital periods in SFXTs range from 3 to 30 days (IGR J16479-4514: 3.32 days, IGR J17544-2619: 4.92 days; IGR J18483-0311：18.5 days; SAX J1818.6-1703: 30.0 days; Jain et al. 2009; Clark et al. 2009; Bird et al. 2009; Zurita Heras \& Chaty 2009; Sguera et al. 2007), and are thus similar to those 
of other SGXBs. The only exception is the SFXT IGR J112155952, which has an orbital period of 165 days (Sidoli et al. 2007). The relatively high eccentricities inferred for two SFXTs ( 0.3-0.7, Zurita Heras \& Chaty 2009; Rahoui et al. 2008) suggest that these systems might be characterized by somewhat more elongated orbits than classical SGXBs. The spin period of the NS hosted in these sources has been measured only in four cases, the periods ranging from 4.7 to $228 \mathrm{~s}$ (IGR J1841.00536: 4.7 s; IGR J1843-0311: 21 s; IGR J16465-4507: 228 s; IGR J11215-5952: 186.78 s; Bamba et al. 2001; Sguera et al. 2007; Lutovinov et al. 2005; Swank et al. 2006), and thus being similar to the spin periods measured in the classical SGXBs. However, owing to the limited duration of most observations, it is not possible to exclude that a number of SFXTs have much longer spin periods (see e.g., Smith et al. 1998; Bozzo et al. 2008).

A number of pointed XMM-Newton observations of several SFXTs were carried out to study the quiescent emission of these sources and gain insight into the mechanism that drives their peculiar X-ray activity. During the observation of the SFXT IGR J16479-4514, XMM-Newton captured the source undergoing a very steep luminosity decay from the end of an outburst to a much lower state. The latter was interpreted in terms of an eclipse of the source by the companion star (Bozzo et al. 2008, 2009; Jain et al. 2009). This observation revealed that in at least one case the X-ray variability of a SFXT was due to the obscuration by the companion star. In the case of IGR J184830311, XMM-Newton helped identify pulsations in the quiescent $X$-ray flux of this source (Giunta et al. 2009), and thus provided strong support for the idea that SFXTs also accrete matter during their quiescent states (see e.g., Sidoli et al. 2007; Bozzo et al. 2008).

To study the low level emission of SFXT sources, we present in this paper quiescent state XMM-Newton observations of the prototypical SFXTs XTE J1739-302 and IGR J08408-4503. In Sect. 2, we summarize previous observations of these sources, and in Sects. 3 and 4 we present our data analysis and results. In particular, we find that the quiescent spectra of these sources contain a soft component below $\sim 2 \mathrm{keV}$. We discuss some possible interpretations of this component in Sect. 5. A comparison is also carried out between the quiescent and outburst spectral properties of XTEJ1739-302 and IGR J08408-4503. Our conclusions are summarized in Sect. 6.

\section{The sources}

\subsection{XTEJ1739-302}

XTE J1739-302 is a SFXT prototype, and was discovered with RXTE during a bright outburst in 1997 (Smith et al. 1998). The identification of its supergiant companion led to the determination of the source distance at $2.7 \mathrm{kpc}$ (Rahoui et al. 2008). Several outbursts from this source were detected later with RXTE (Smith et al. 2006), and INTEGRAL (Lutovinov et al. 2005; Sguera et al. 2005, 2006; Blay et al. 2008). XTE J1739-302 was observed in outburst with Swift/BAT on three occasions, on 2008 April 8 (Sidoli et al. 2009a), on 2008 August 13 (Sidoli et al. 2009b), and on 2009 March 10 (Romano et al. 2009c). In only the first two cases, Swift slewed to the source and observations with the X-ray Telescope, XRT, were carried out. During the 2008 April 8 outburst, XRT observed XTE J1739-302 387 s after the BAT trigger. These data showed that the source was rapidly $(\sim 1000 \mathrm{~s})$ decreasing in intensity, and the X-ray spectrum $(0.3-10 \mathrm{keV})$ could be reproduced well by using an absorbed $\left(N_{\mathrm{H}}=13 \times 10^{22} \mathrm{~cm}^{-2}\right)$ power law (hereafter, PL) model (photon index $\Gamma=1.5$ ). The $0.5-100 \mathrm{keV} X$-ray luminosity was $\sim 3.0 \times 10^{36} \mathrm{erg} \mathrm{s}^{-1}$. Sidoli et al. (2009a) also performed an analysis of the Swift broad band (0.3-60 keV) spectrum of XTE J1739-302 during this outburst, and found that this spectrum could be reasonably well described by using either a power law with a cutoff at high energy $(\sim 13 \mathrm{keV})$, or a Comptonizing plasma model (COMPTT in XSPEC). For the outburst of 2008 August 13, XRT data were obtained starting from $\sim 390 \mathrm{~s}$ after the BAT trigger, and revealed a more complex behavior than that observed during the previous event (Sidoli et al. 2009b). A time-resolved analysis showed that the source X-ray spectrum could be fit equally well by using an absorbed PL or a black-body (BB) model with constant photon index or temperature $\left(\Gamma \sim 1.2, k T_{\mathrm{BB}} \sim 1.8 \mathrm{keV}\right)$, and a varying absorption column density (in the range $3-9 \times 10^{22} \mathrm{~cm}^{-2}$ ). The combined XRT+BAT broad band (0.3-60 keV) spectrum could be well fit by using either a model of Comptonization of seed photons in a hot plasma (COMPTT in XSPEC) or a BMC model. The BMC comprises a BB component and a component accounting for the Comptonization of the $\mathrm{BB}$ due to thermal and/or dynamical (bulk) Comptonization. The $0.1-100 \mathrm{keV}$ X-ray luminosity derived from the simultaneous XRT+BAT spectrum was $\sim 3.8 \times 10^{36} \mathrm{erg} \mathrm{s}^{-1}$. On 2009 March 10, XTE J1739-302 again triggered BAT (Romano et al. 2009c). On this occasion, Swift did not perform any quick slew towards the source and XRT data were accumulated only $\sim 1.5 \mathrm{~h}$ after the BAT trigger. At this time, the source was already much fainter (X-ray luminosity $\sim 7 \times 10^{34} \mathrm{erg} \mathrm{s}^{-1}, 2-10 \mathrm{keV}$ ), and the XRT spectrum could be reproduced well by using an absorbed power-law model $\left(N_{\mathrm{H}}=4 \times 10^{22} \mathrm{~cm}^{-2}, \Gamma=1.2\right)$.

Little is known about the quiescent emission of XTE J1739302. An ASCA observation in 1999 did not detect the source and placed a $3 \sigma$ upper limit on its X-ray luminosity of $8 \times 10^{32} \mathrm{erg} \mathrm{s}^{-1}$ (exposure time $\sim 13 \mathrm{ks}$, Sakano et al. 2002). A $\sim 5 \mathrm{ks}$ Chandra observation in 2001 caught the source in a relatively low luminosity state $\left(9.7 \times 10^{33} \mathrm{erg} \mathrm{s}^{-1}\right)$ and the $\mathrm{X}$-ray spectrum was fit well by using an absorbed power-law model $\left(N_{\mathrm{H}} \sim 4.2 \times\right.$ $10^{22} \mathrm{~cm}^{-2}, \Gamma=0.62$; Smith et al. 2006). Based on a monitoring program with Swift, Romano et al. (2009b) carried out the first study of the long-term variation in the quiescent emission from XTE J1739-302. They identified three different quiescent states of the source, with a $2-10 \mathrm{keV} X$-ray luminosity of $10^{35}, 2 \times 10^{34}$, and $5 \times 10^{32} \mathrm{erg} \mathrm{s}^{-1}$, respectively. The Xray spectra of these states could be reasonably well described by using an absorbed power-law model, with absorption column densities and photon indices in the range $(0.3-3.6) \times 10^{22} \mathrm{~cm}^{-2}$ and $0.5-1.6$, respectively. Alternatively, these spectra could also be fit by using a BB model with temperatures and radii in the range $1.3-1.9 \mathrm{keV}$ and $0.02-0.28 \mathrm{~km}$, respectively. With these results at hand, the authors argued that the quiescent emission of XTE J1739-302 was most likely produced by a spot covering a relatively small region of the NS surface, possibly the NS magnetic polar caps.

\subsection{IGR J08408-4503}

IGR J08408-4503 was discovered in the Vela region on 2006 May 15 with INTEGRAL during a short flare lasting less than $1000 \mathrm{~s}$ (Götz et al. 2007). Its optical counterpart was later identified as the supergiant star HD 74194 located at $3 \mathrm{kpc}$, thus confirming that this source belongs to the SFXT class (Götz et al. 2007; Masetti et al. 2006). IGR J08408-4503 was observed in outburst two times with INTEGRAL, and the combined JEM-X and ISGRI spectra were most accurately fit by using a 
XTE 31 1139-302 (EPic-PN OBS1)

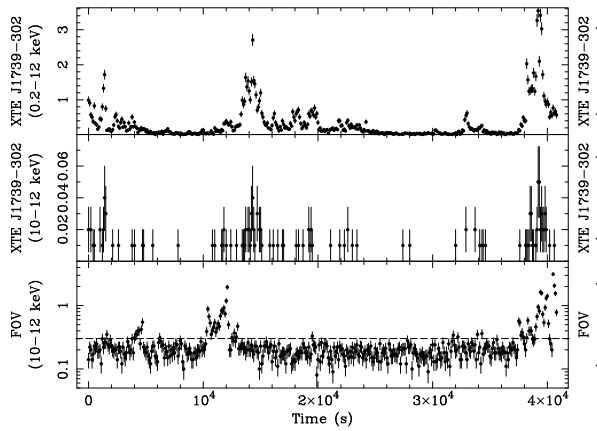

XTE J1\%39-302 (EPic-PN OBS2)

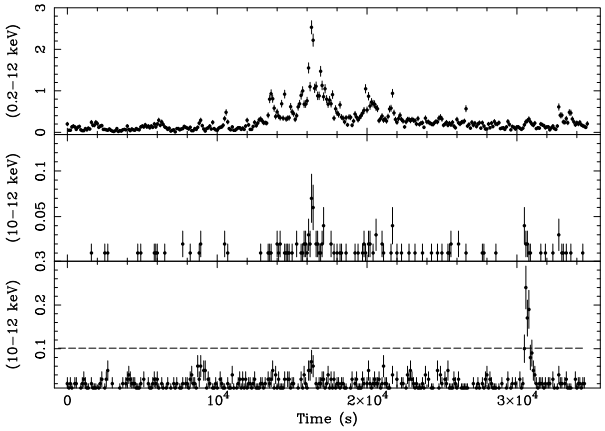

IGR J08408-4503 (EPic-PN)

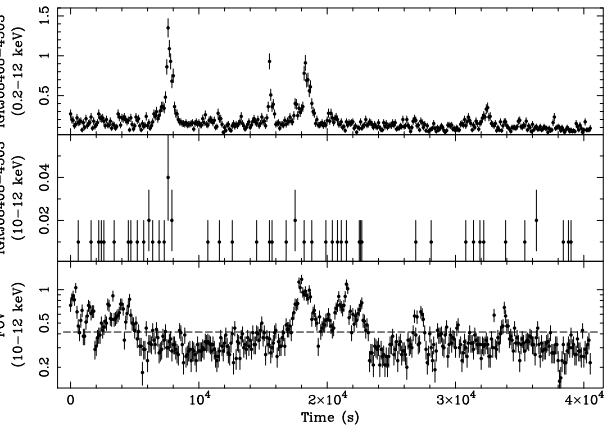

Fig. 1. Selection of the high background intervals during the two XMM-Newton observations (Epic-PN camera) of XTE J1739-302 (OBS1 on the left and OBS2 in the middle) and the observation of IGR J08408-4503 (on the right). In each case, we reported the source lightcurve not corrected for the selection of the good time intervals and not subtracted for the background in the 0.2-12 keV (upper panel) and 10-12 keV energy band (middle panel), and the count rate of the total FOV in the 10-12 keV energy band (lower panel). In all cases, the time bin is $100 \mathrm{~s}$. Only the observational intervals in which the total FOV count rate in the 10-12 keV energy band was below the threshold indicated with a dashed line were considered for the timing and spectral analysis of the sources.

rather flat cut-off power-law model $\left(\Gamma \simeq 0, E_{\text {cut }} \sim 11-15 \mathrm{keV}\right.$, Götz et al. 2007; Leyder et al. 2007). The absorption column density was $\sim 0.1 \times 10^{22} \mathrm{~cm}^{-2}$, compatible with the interstellar value in the direction of the source (Dickey \& Lockman 1990). The $0.1-100 \mathrm{keV}$ luminosity of the two outbursts was $7.6 \times 10^{35}$ and $3 \times 10^{36} \mathrm{erg} \mathrm{s}^{-1}$.

IGR J08408-4503 was also caught in outburst by Swift 4 times between 2006 and 2009 (Romano et al. 2009a; Sidoli et al. 2009b; Barthelmy et al. 2009). During the first outburst, which occurred on 2006 October 4, Swift/XRT slewed to the source $\sim 2000 \mathrm{~s}$ after the BAT trigger. The combined XRT+BAT spectrum could be reproduced well by using a cut-off powerlaw model $\left(\Gamma=0.31, E_{\mathrm{c}} \simeq 11 \mathrm{keV}\right)$. The column density was $\sim 0.3 \times 10^{22} \mathrm{~cm}^{-2}$. The second outburst, which took place on 2008 July 5, was characterized by complex behavior and comprised two different flares separated by $\sim 10^{5} \mathrm{~s}$. The timeresolved spectral analysis found that the soft X-ray emission of the source could be reasonably well described by using an absorbed power-law model with a constant photon index of $\sim 1$ and a variable absorption column density ranging from $\sim 0.5$ to $\sim 11 \times 10^{22} \mathrm{~cm}^{-2}$. The simultaneous XRT+BAT broad band $(0.3-$ $80 \mathrm{keV}$ ) spectrum of the source could be reproduced well using an absorbed cut-off power-law model, with $E_{\mathrm{c}} \gtrsim 14 \mathrm{keV}$, $\Gamma \sim 1.4$, and $N_{\mathrm{H}}=6.7 \times 10^{22} \mathrm{~cm}^{-2}$. The third outburst occurred on 2008 September 21. On this occasion, Swift/XRT slewed to the source $\sim 150 \mathrm{~s}$ after the BAT trigger, and time-resolved spectroscopy did not reveal any variation in the absorption column density. However, the total XRT spectrum of the observation (exposure time $1160 \mathrm{~s}$ ) clearly revealed a previously undetected soft component below $\sim 2 \mathrm{keV}$. A reasonably good fit to these data could be obtained by using an absorbed $\left(N_{\mathrm{H}}=0.4 \times 10^{22} \mathrm{~cm}^{-2}\right)$ power-law $(\Gamma \simeq 2.2)$ component and a $\mathrm{BB}$ at the softer energies (with a temperature of $k T \sim 1.95 \mathrm{keV}$, and a radius of $\sim 1.2 \mathrm{~km}$ ). This BB component was also required to fit the combined XRT+BAT broad band spectrum of the outburst (a single comptonization model, BMC, only provided a poor fit to the data). Sidoli et al. (2009b) found that the seed photon temperature of the BMC component and the BB temperature could not be linked to the same value in the fit. This was interpreted as being caused by the presence of two distinct photon populations, a colder one with a temperature of about $0.3 \mathrm{keV}$ and a radius of $\sim 10 \mathrm{~km}$, and a hotter one with a temperature of $1.4-1.8 \mathrm{keV}$ and a radius of $\sim 1 \mathrm{~km}$. However, the statistics of the data did not allow the authors to distinguish which of these two populations was seen directly as a BB and which one ended up being seed photons for the thermal Comptonization. The latest outburst from IGR J08408-4503 was caught by Swift/BAT on 2009 August 28. Unfortunately, in this case Swift/XRT did not perform any follow-up observation, and thus no detailed spectral or timing information was available in the $0.3-10 \mathrm{keV}$ energy band.

To date, the quiescent emission of IGR J08408-4503 has remained largely unexplored; the only detection of this source during a period of low X-ray activity was obtained by two Swift/XRT follow-up observations carried out in 2006 May 22 and 2007 September 29 (Leyder et al. 2007). The exposure time of each of these observations was about $\sim 4.0 \mathrm{ks}$, and only a total of 40 photons could be collected. Assuming a Crab-like spectrum, the source X-ray flux was estimated to be $\sim 2 \times 10^{-13} \mathrm{erg} \mathrm{cm}^{-2} \mathrm{~s}^{-1}(0.5-10 \mathrm{keV})$, corresponding to a luminosity of about $2 \times 10^{32} \mathrm{erg} \mathrm{s}^{-1}$ (Kennea \& Campana 2006).

\section{XMM-Newton data analysis}

For the present study, we used two XMM-Newton observations of XTE J1739-302, and one XMM-Newton observation of IGR J08408-4503. During all three observations, the two sources were in quiescence.

XMM-Newton observation data files (ODFs) were processed to produce calibrated event lists using the standard XMM-Newton Science Analysis System (v. 9.0). We used the EPPROC and EMPROC tasks for the Epic-PN and the two MOS cameras, respectively. The event files of the two observations were filtered to exclude high background time intervals. The effective exposure time for each observation and camera is given in Sects. 4.1 and 4.2. Source lightcurves and spectra were extracted in the $0.2-15 \mathrm{keV}$ band for the Epic-PN and $0.2-10 \mathrm{keV}$ for the two Epic-MOS cameras ${ }^{1}$. We extracted the background lightcurves and spectra from the nearest source-free region to XTE J1739-302 and IGR J08408-4503. Background and source circles were all chosen to lie within the same CCD. The difference in extraction areas between source and background was accounted for by using the SAS BACKSCALE task for the spectra and the LCMATH task from HEASOFT for the lightcurves. All of the EPIC spectra were rebinned before fitting so as to have at least 25 counts per bin and, at the same time, prevent oversampling the energy resolution by more than a factor of three. Given the low count rate of the source, the Epic-MOS1 and Epic-MOS2

\footnotetext{
1 See http://xmm2.esac.esa.int/docs/documents/ CAL-TN-QQ18.pdf.
} 
XTEJ1739-302 (Epic-PN OBS1)

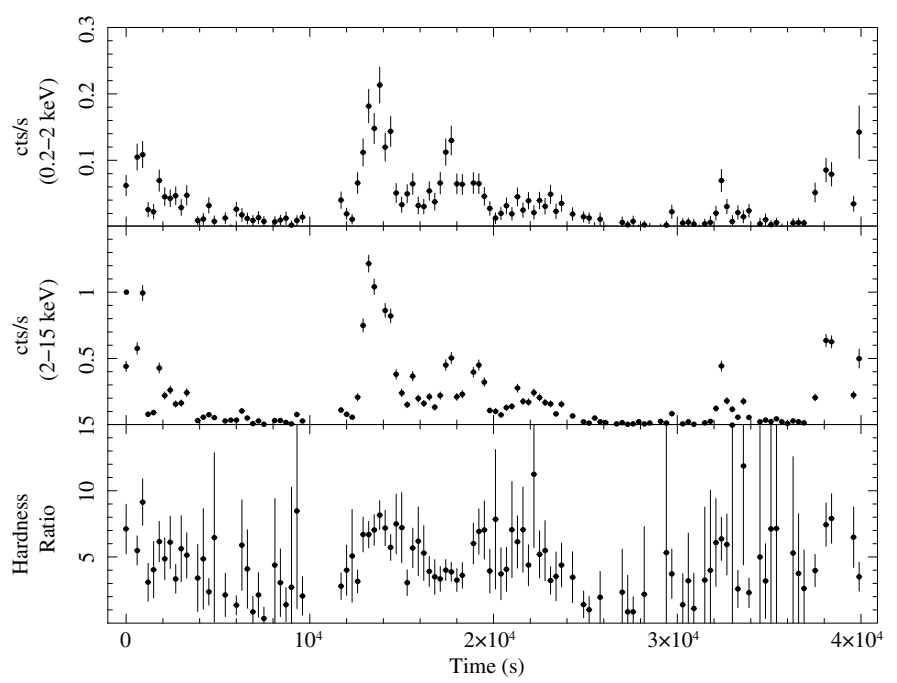

Fig. 2. XMM-Newton Epic-PN background-subtracted lightcurve of XTE J1739-302 during the observation carried out on 2008 October 1. The upper panel shows the source lightcurve in the $0.2-2 \mathrm{keV}$ energy band, whereas the middle panel gives the lightcurve in the $2-15 \mathrm{keV}$ energy band (the binning time is $300 \mathrm{~s}$ ). The ratio of the source count rate in the two bands, $(2-15 \mathrm{keV}) /(0.2-2 \mathrm{keV})$, versus time is shown in the lower panel. The time intervals in which no data are plotted have been discarded because of high background events.

cameras did not contribute significantly to the spectral analysis; we therefore discuss in this paper only the spectra from the Epic-PN camera. Throughout this paper, the errors are given at $90 \%$ c.l. (unless stated otherwise).

\section{Results}

\subsection{XTEJ1739-302}

XMM-Newton observed XTEJ1739-302 twice, on 2008 October 1 (hereafter OBS1) and on 2009 March 11 (hereafter OBS2). The Epic-PN camera was operated in large window mode in the first case and in small window mode during the second observation. To identify the high background time intervals, we followed the SAS online analysis thread ${ }^{2}$ and extracted the Epic-PN lightcurves for the full field of view (FOV) in the 10-12 keV energy band. We discarded time intervals in OBS1 and OBS2 when the 10-12 keV FOV count rate was higher than 0.3 and $0.1 \mathrm{cts} / \mathrm{s}$, respectively. This is illustrated in Fig. 1, where we report the source lightcurves (uncorrected for the background and the good time-interval selection) in the energy band $0.2-12 \mathrm{keV}$ (upper panel), the contribution of the source during the two observations in the energy band 10-12 keV (middle panel), and the count rate of the total FOV in the energy band 10-12 keV (lower panel). The threshold we imposed in each case is plotted with a dashed line in the bottom panel. After the good time intervals were selected, we obtained a total effective exposure time of $32 \mathrm{ks}$ and $24 \mathrm{ks}$ for OBS1 and OBS2, respectively.

The lightcurves of the two observations corrected for the good time interval selection and background-subtracted are shown in Figs. 2 and 3. In both cases, the source displayed a pronounced variability on a timescale of hundreds of seconds,

\footnotetext{
2 See also http: //xmm.esac.esa.int/sas/current/ documentation/threads/PN_spectrum_thread.shtml.
}

XTEJ1739-302 (Epic-PN OBS2)

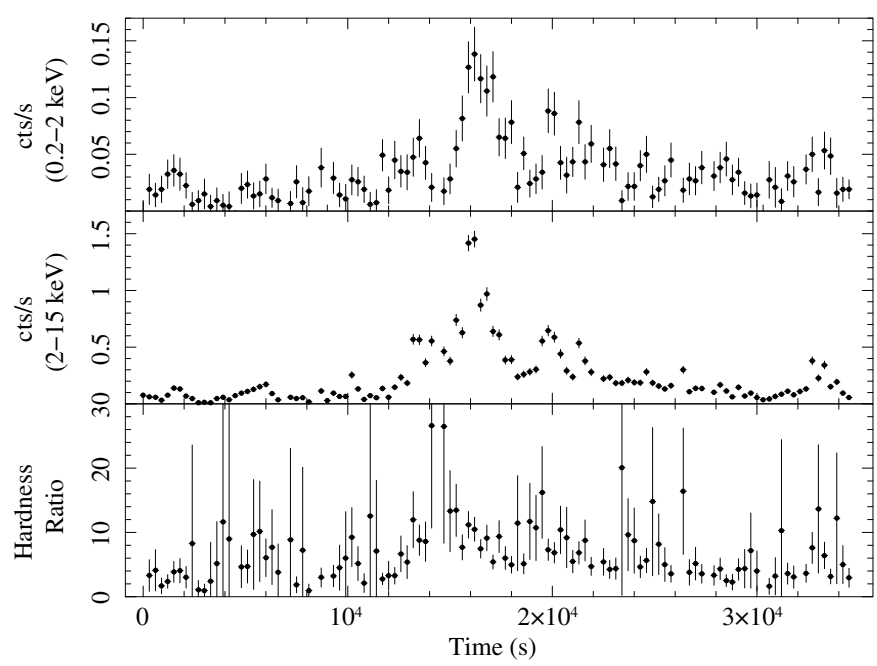

Fig. 3. The same as Fig. 2, but for the XMM-Newton observation carried out on 2009 March 11. The binning time is $300 \mathrm{~s}$.
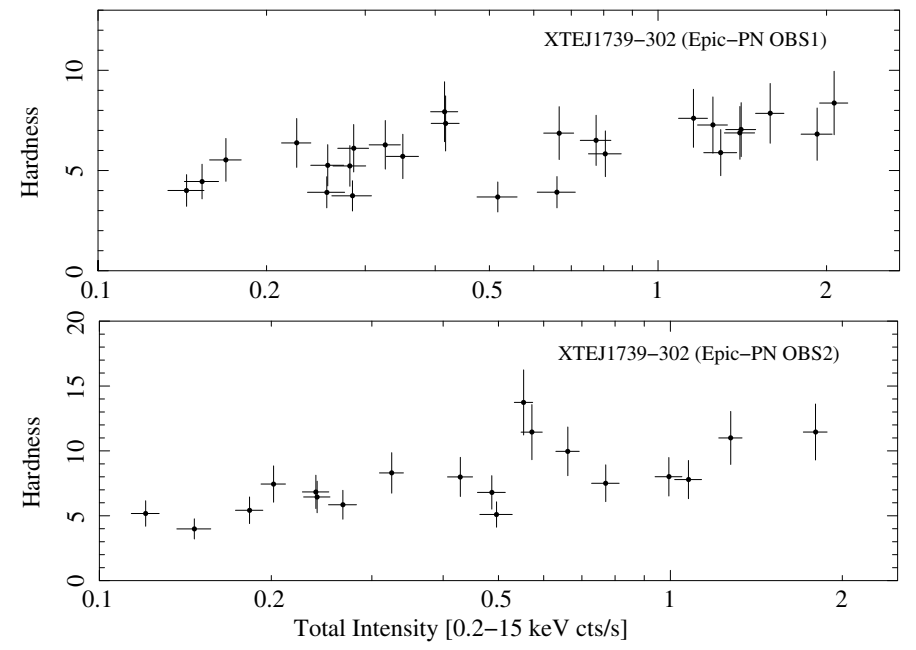

Fig. 4. Hardness-intensity diagram for the two XMM-Newton observations of XTE J1739-302 (the upper panel is for the observation carried out in 2008, the lower panel for the observation in 2009). The hardness is defined as the ratio of the count rate in the hard $(2-15 \mathrm{keV})$ to soft $(0.2-2 \mathrm{keV})$ energy band. The points were obtained by using the lightcurves given in Figs. 2 and 3, but, wherever necessary, consecutive bins were rebinned so as to achieve a $S / N>5.5$ in the hardness ratio.

Table 1. Results of the count-rate-resolved spectral analysis for the two XMM-Newton observations of XTE J1739-302.

\begin{tabular}{lllllll}
\hline \hline & \multicolumn{5}{c}{ OBS1 } & \multicolumn{3}{c}{ OBS2 } \\
cts/s & $<0.1$ & $0.1-0.4$ & $>0.4$ & $<0.1$ & $0.1-0.4$ & $>0.4$ \\
\hline$N_{\mathrm{H}}{ }^{a}$ & $2.9_{-0.6}^{+0.5}$ & $2.7 \pm 0.3$ & $2.6 \pm 0.3$ & $4.1_{-0.6}^{+0.7}$ & $3.2_{-0.2}^{+0.3}$ & $3.5 \pm 0.4$ \\
$\Gamma$ & $1.8 \pm 0.3$ & $1.4 \pm 0.1$ & $1.1 \pm 0.1$ & $1.8 \pm 0.3$ & $1.2 \pm 0.1$ & $1.0 \pm 0.1$ \\
$F_{\text {obs }}{ }^{b}$ & $4.7_{-3.2}^{+0.9}$ & $32.2_{-7.1}^{+3.4}$ & $123.8_{-20.7}^{+10.0}$ & $9.3_{-6.9}^{+1.2}$ & $42.0_{-7.6}^{+4.5}$ & $153.6_{-26.5}^{+13.7}$ \\
$\chi_{\text {red }}^{2}$ & 1.08 & 1.11 & 1.00 & 0.74 & 1.04 & 1.00 \\
d.o.f. $^{c}$ & 33 & 93 & 105 & 34 & 132 & 89 \\
EXP. $^{c}$ & 18 & 10 & 3 & 10 & 11 & 2 \\
\hline
\end{tabular}

Notes. The model used to fit the data is an absorbed CUTOFFPL (we fixed the cutoff energy at $13 \mathrm{keV}$, see text for details). (a) in units of $10^{22} \mathrm{~cm}^{-2}$. ${ }^{(b)}$ Observed flux in the $0.5-10 \mathrm{keV}$ energy band in units of $10^{-13} \mathrm{erg} \mathrm{cm}^{-2} \mathrm{~s}^{-1}$. ${ }^{(c)}$ Exposure time in ks. 
XTEJ1739-302 (Epic-PN OBS1)

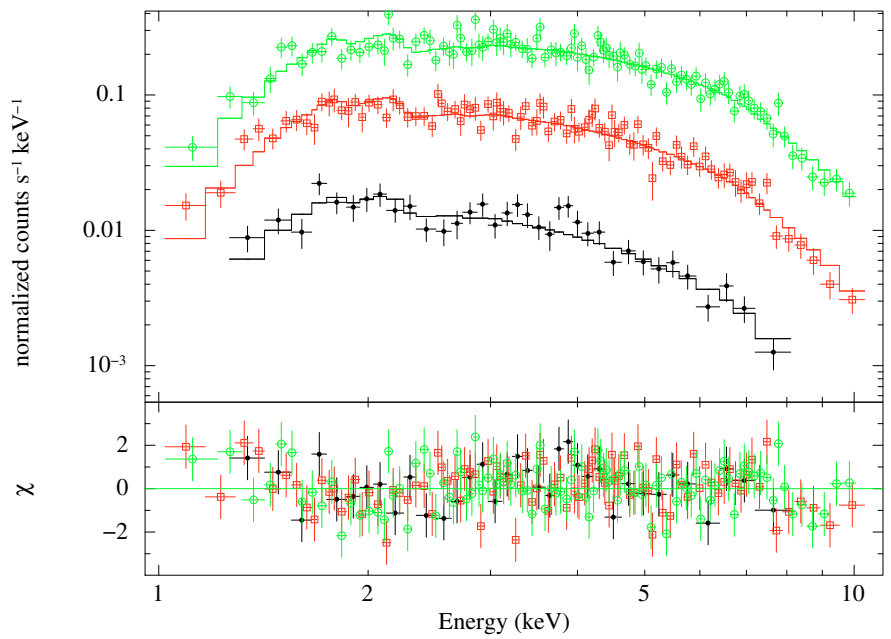

Fig. 5. The XMM-Newton spectra of the XTEJ1739-302 observation carried out in 2008. The open circles, open squares, and filled circles are from the energy resolved data obtained during the time intervals in which the Epic-PN 0.2-15 keV source count rate was $>0.4,0.1-$ $0.4,<0.1$, respectively. The model used for the best fits is an absorbed CUTOFFPL (cutoff energy fixed at $13 \mathrm{keV}$ ). The residuals from this fit are shown in the bottom panel. (See the electronic edition of the paper for a color version of this figure.)

XTEJ1739-302 (Epic-PN OBS2)

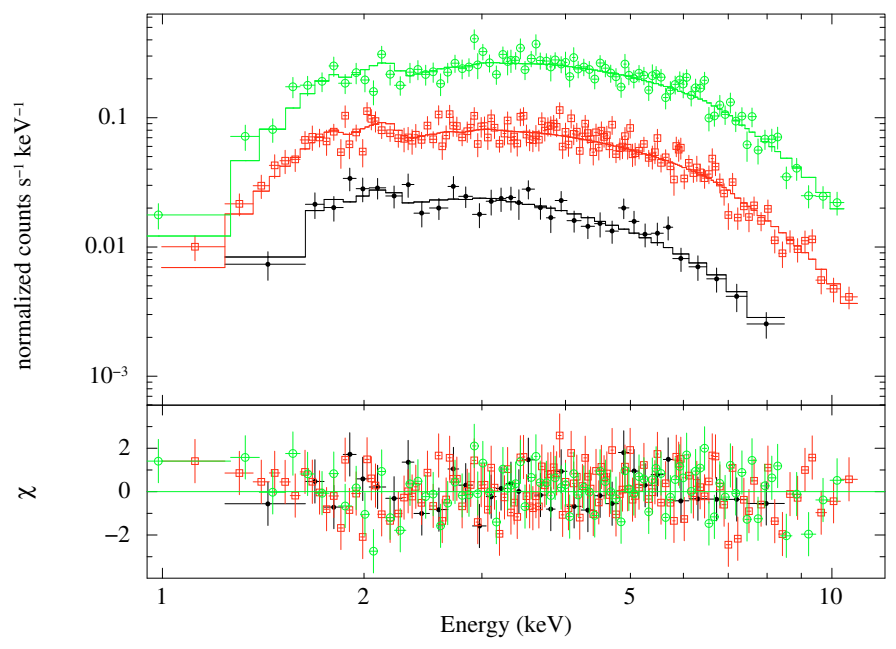

Fig. 6. The same as Fig. 5 but for the observation carried out in 2009. The model used for the best fits is an absorbed CUTOFFPL (cutoff energy fixed at $13 \mathrm{keV}$ ). The residuals from these fits are shown in the bottom panel. (See the electronic edition of the paper for a color version of this figure.)

with small flares occurring sporadically after periods of lower $\mathrm{X}$-ray emission. During these flares, the X-ray flux typically increased by a factor of $\sim 10-30$ (from a few $10^{-13} \mathrm{erg} \mathrm{cm}^{-2} \mathrm{~s}^{-1}$ up to $\sim 10^{-11} \mathrm{erg} \mathrm{cm}^{-2} \mathrm{~s}^{-1}$ ). From the lower panels in Figs. 2 and 3, it is apparent that the source emission hardened at higher count rates. To investigate this behavior in more detail, we plot in Fig. 4 the hardness ratio of the source (i.e. the ratio of the source count rate in the hard, $2-15 \mathrm{keV}$, to the soft, $0.2-2 \mathrm{keV}$, energy bands) as a function of the total intensity (i.e. the total source count rate in the $0.2-15 \mathrm{keV}$ energy band). For these plots, we rebinned the lightcurves of Figs. 2 and 3 to obtain a $S / N \geq 5.5$. The scatter in
XTEJ1739-302 (Epic-PN OBS1)

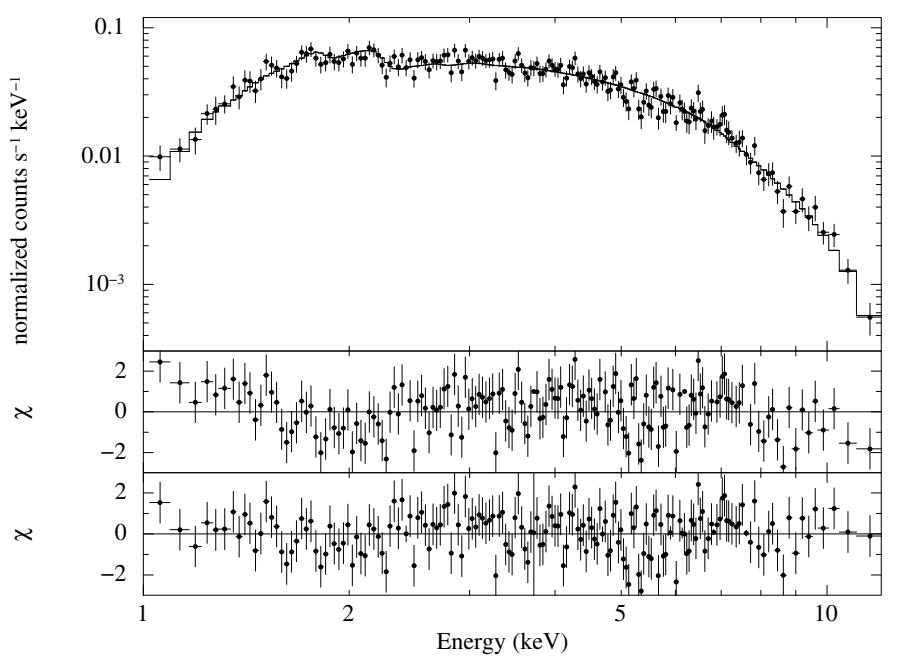

Fig. 7. The XMM-Newton spectrum of XTE J1739-302 extracted by using the total exposure time of OBS1. The model used for the best fit is an absorbed CUTOFFPL (we did not fix here the cutoff energy). The residuals from this fit are shown in the bottom panel, whereas the middle panel shows the residuals from the fit obtained by using an absorbed CUTOFFPL with the cutoff energy fixed at $13 \mathrm{keV}$.

the points in Fig. 4 suggests that the relation between the source count rate and the hardness is most likely changing from flare to flare. However, an overall increase in the hardness with the source intensity is apparent from the plots. To test this correlation, we performed a linear regression that accounts for the errors in both variables (Press 2002). The slope of the linear function determined from this analysis is $1.9 \pm 0.4$ and $4.1 \pm 0.8$ for OBS1 and OBS2, respectively (errors are at $1 \sigma$ c.1.), thus confirming that the correlation is significant $(\sim 5 \sigma)$. To investigate which spectral parameters varied with source intensity, we divided the lightcurves of the two observations into three intensity levels, and accumulated the spectra during the corresponding time intervals. We selected the intervals in which the $0.2-15 \mathrm{keV}$ Epic-PN count rate of XTE J1739-302 was < 0.1, 0.1-0.4, and $>0.4$, for both OBS 1 and OBS2. The three spectra extracted from each observation were then fitted together with an absorbed cutoff power-law model (hereafter CUTOFFPL, see Figs. 5 and 6). We fixed the cutoff energy at $13 \mathrm{keV}$ (Sidoli et al. 2009a), and found that this model provided a closer fit to the data than a simple absorbed PL model. We report the results of the fit with the absorbed CUTOFFPL model in Table 1 (we also attempted to fit the data with an absorbed BB model, but this gave a poorer result for some of the rate-resolved spectra, with $\chi^{2} \gtrsim 1.2$ ). For OBS1, the rate-resolved spectral analysis demonstrated that the power-law photon index changed from $1.1 \pm 0.1$, in the highest source count-rate spectrum, to $1.8 \pm 0.3$, in the spectrum corresponding to the lowest source count rate. No significant variation in the absorption column density was measured, and the best fit value of $N_{\mathrm{H}}$ was consistent (to within the errors) with being constant at a level of $\sim 2.6 \times 10^{22} \mathrm{~cm}^{-2}$. A similar analysis carried out for OBS2 yielded very similar results. In this case, the power-law photon index decreased at the higher source count rate from $1.8 \pm 0.3$ to $1.0 \pm 0.1$, and the absorption column density remained constant (to within the errors) at a value of $\sim 4.0 \times 10^{22} \mathrm{~cm}^{-2}$. As the measured changes in the spectral parameters were not dramatic, we also extracted the X-ray spectra of the source by using all the data from each observation. 


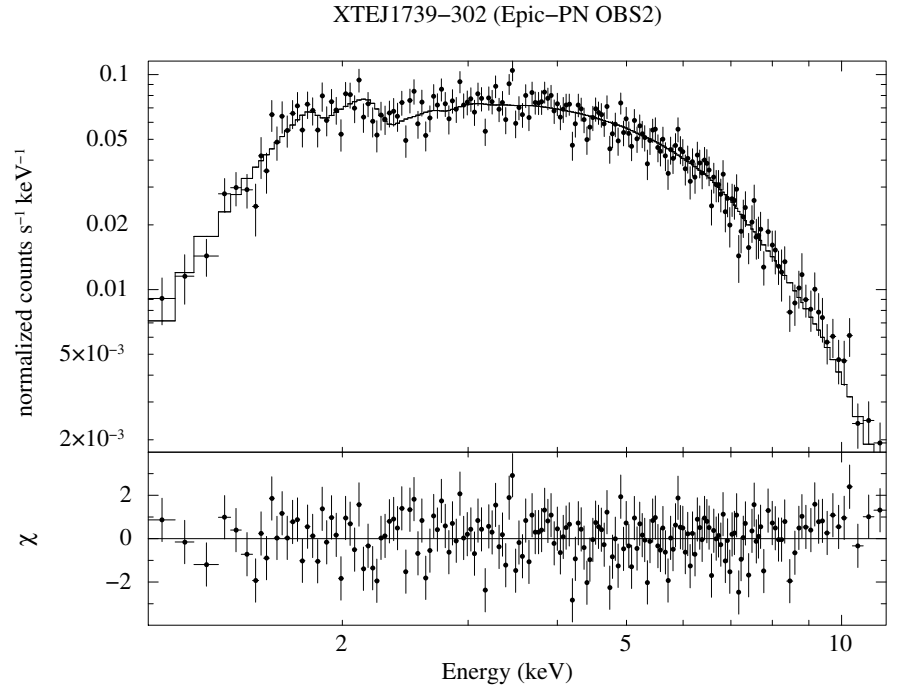

Fig. 8. The same as Fig. 7, but for the observation carried out on 2009 March 11. Here the best-fit model to the data was an absorbed CUTOFFPL model (the cutoff energy was allowed to vary during fitting).

XTEJ1739-302 (Epic-PN OBS1)

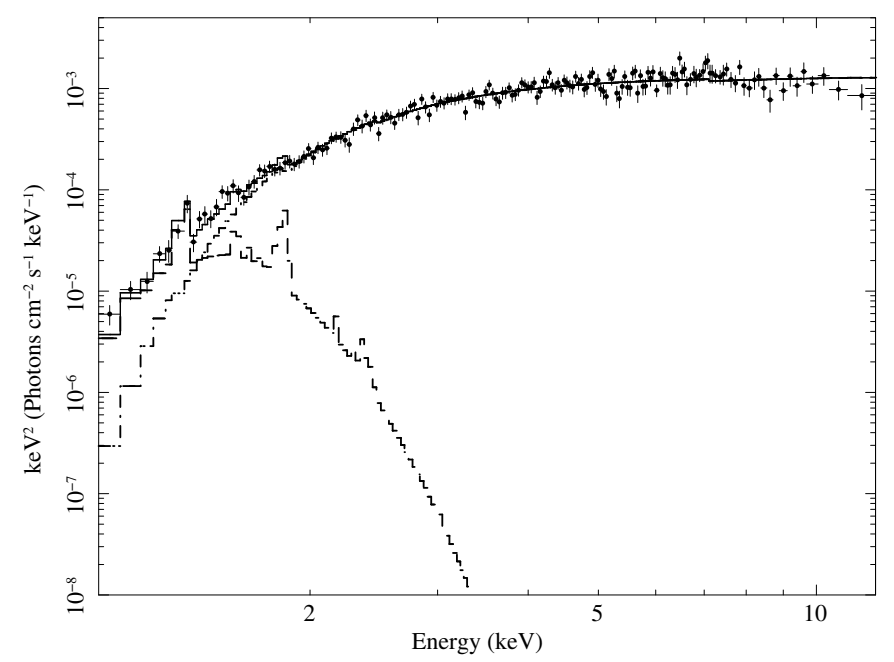

Fig. 9. An example of an unfolded spectrum of the XMM-Newton observation of XTE J1739-302 carried out in 2008 (OBS1). Here the best fit is obtained by using an absorbed CUTOFFPL plus a MKL component (see Sect 4.1).

These spectra are shown in Figs. 7 and 8. A fit to these spectra with a single absorbed PL or BB model gave a relatively poor $\chi_{\text {red }}^{2}(\gtrsim 1.3$, d.o.f. $=175,184$ for OBS1 and OBS2, respectively) fit, and a slightly better result was obtained using the same CUTOFFPL model discussed before for the rate-resolved spectra (cutoff energy fixed at $13 \mathrm{keV}, \chi_{\text {red }}^{2} \lesssim 1.2$, d.o.f. $=175,184$ for OBS1 and OBS2, respectively). However, even in this case, the reduced $\chi^{2}$ remained significantly higher than 1 and, especially in OBS1, some structures are apparent in the fit residuals (see Fig. 7).

By leaving the cutoff energy free to vary in the fit, a significantly closer fit was obtained, with a $\chi_{\text {red }}^{2} /$ d.o.f. $=1.02 / 174$, 1.09/183 for OBS1 and OBS2, respectively. By using the F-test, we found that this improvement was highly significant for OBS1 $(5.3 \sigma)$ and somewhat less significant for OBS2 $(3.5 \sigma)$. In both cases, the derived cutoff energy turned out to be much lower ( $\sim 4 \mathrm{keV}$, see Table 2 ) than that measured previously when the source was in outburst $(\sim 13 \mathrm{keV})$. Such a low value for the cutoff energy might not be unlikely for an X-ray pulsar (see Sect. 5). In the case of OBS1, when an additional spectral component to that of the CUTOFFPL appears to be clearly significant, we also tried to investigate the applicability of other spectral models. We again fixed the cutoff energy of the CUTOFFPL component at $13 \mathrm{keV}$ and tried first a phenomenological model including an additional black-body component (BB) at lower energies $(<2 \mathrm{keV})$. We found that both a BB with $k T \sim 1-2 \mathrm{keV}$ and a small emitting area (few hundreds $\mathrm{m}^{2}$ ) and a much colder ( $k T \sim 0.1-0.3 \mathrm{keV}) \mathrm{BB}$ with an emitting radius of $\sim 100 \mathrm{~km}$ could reproduce the spectrum reasonably well. The interpretation of both of these models for the soft X-ray excess faces difficulties: a BB emitting area of about one hundred $\mathrm{km}$ radius would be much larger than the NS, while a small emitting spot on the star surface might also be unlikely in the case of a low-luminosity wind-accreting NS (see Sect. 5). We therefore tried to fit the spectrum of OBS1 using models that provide to alternative physical interpretations. We adopted first a model comprising a CUTOFFPL and a MKL component, and then tried a second model in which we included the effect of partial covering (ZPCFABS in XSPEC) on the CUTOFFPL component. We note that these two models were suggested to fit the quiescent XMM-Newton spectra of the SFXTs AX J1845-0433 (Zurita Heras \& Walter 2009) and IGR J16207-5129³ (Tomsick et al. 2009). In these cases, the authors suggested that the MKL component might represent the contribution due to the shocks in the stellar wind around the NS, whereas the partial covering may be caused by the obscuration of the NS by clumps in this wind. Finally, we also attempted to fit the spectrum of OBS1 by using the COMPTT and the BMC models, as suggested by Sidoli et al. (2009a) and Sidoli et al. (2009b). These two models provided a good fit to the data, but in the case of the BMC we found that most of the model parameters were only poorly determined in the fit, and thus we do not discuss this model further in the case of XTEJ1739-302. The results of the fits obtained with all the other models discussed in this section are reported in Table 2. We checked that a fit to the spectrum of OBS2 with all these models would give results comparable to those obtained for OBS1. All the spectral models reported in Table 2 provided reasonable values of the fit parameters and very similar $\chi_{\text {red }}^{2}$ (note that all the models have the same number of free parameters). In the best case, we obtained $\chi_{\text {red }}^{2} /$ d.o.f. $=0.98 / 173$, and thus the significance of the improvement of these fits, with respect to that obtained with a simple absorbed CUTOFFPL model and the energy cutoff fixed at $13 \mathrm{keV}$, was of $5.6 \sigma$. As an example, we show in Fig. 9 the unfolded spectrum of OBS1 obtained by using a MKL component to fit the low-energy excess. The interpretation of these results is discussed in Sect. 5.

We also performed a Fourier analysis of the lightcurves of OBS1 and OBS2, in search of coherent pulsations, by using the method described in Israel \& Stella (1996). No significant (above $3 \sigma$ level) signal was detected in either observation. The corresponding $3 \sigma$ c.l. upper limits to the pulsed fraction (defined as the semi-amplitude of the sinusoid divided by the source average count rate), were then computed according to the method described in Vaughan et al. (1994). In OBS1, upper limits at a level of $30 \%, 20 \%$, and $40 \%$ were inferred in the $0.1-0.2 \mathrm{~s}, 0.2-50 \mathrm{~s}$, and $50-150$ s period range, respectively. In OBS2, we derived

\footnotetext{
3 This source was classified as an intermediate SFXT by Walter \& Zurita Heras (2007).
} 
Table 2. Spectral fits of the XMM-Newton data of XTE J1739-302.

\begin{tabular}{|c|c|c|c|c|c|c|}
\hline \multirow[b]{2}{*}{ Model } & \multicolumn{5}{|c|}{ OBS1 } & \multirow{2}{*}{$\begin{array}{c}\text { OBS2 } \\
\text { CUTOFFPL }\end{array}$} \\
\hline & CUTOFFPL & $\mathrm{BB}+\mathrm{CUTOFFPL}$ & COMPTT & MKL+CUTOFFPL & ZPCFABS*CUTOFFPL & \\
\hline$N_{\mathrm{H}}^{a}$ & $\begin{array}{c}1.9_{-0.3}^{+0.2} \\
\left(2.7_{-0.1}^{+0.2}\right)\end{array}$ & $\begin{array}{c}1.7_{-0.2}^{+0.4} \\
(3.7 \pm 0.4)\end{array}$ & $1.3 \pm 0.2$ & $3.4 \pm 0.4$ & $1.9 \pm 0.4$ & $\begin{array}{c}2.5_{-0.2}^{+0.5} \\
(3.4 \pm 0.2)\end{array}$ \\
\hline$\Gamma$ & $\begin{array}{c}0.2_{-0.4}^{+0.3} \\
(1.3 \pm 0.1)\end{array}$ & $\begin{array}{c}0.8_{-0.6}^{+0.4} \\
(1.5 \pm 0.1)\end{array}$ & - & $1.5 \pm 0.1$ & $1.6 \pm 0.1$ & $\begin{array}{c}0.1_{-0.3}^{+0.6} \\
(1.19 \pm 0.07)\end{array}$ \\
\hline$E_{\text {cut }}(\mathrm{keV})$ & $\begin{array}{c}3.5_{-0.7}^{+0.9} \\
\text { (13 fixed) }\end{array}$ & 13 (fixed) & - & 13 (fixed) & 13 (fixed) & $\begin{array}{c}3.8_{-0.6}^{+2.2} \\
\text { (13 fixed) }\end{array}$ \\
\hline$k T(\mathrm{keV})$ & - & $\begin{array}{c}1.2 \pm 0.1 \\
(0.13 \pm 0.01)\end{array}$ & $0.8 \pm 0.1$ & $0.15 \pm 0.2$ & - & - \\
\hline$R_{\mathrm{BB}}^{b}(\mathrm{~km})$ & - & $\begin{array}{c}0.07 \pm 0.02 \\
\left(43_{-22}^{+42}\right)\end{array}$ & - & - & - & - \\
\hline$k T_{\mathrm{e}}(\mathrm{keV})$ & - & - & $2.8_{-0.7}^{+7.2}$ & - & - & - \\
\hline$\tau$ & - & - & $6.5_{-1.6}^{+2.7}$ & - & - & - \\
\hline$N_{\mathrm{MKL}}$ & - & - & - & $0.3_{-0.2}^{+1.7}$ & - & - \\
\hline$N_{\mathrm{H} 2}{ }^{c}$ & - & - & - & - & $3.6_{-1.0}^{+1.5}$ & - \\
\hline$f$ & - & - & 一- & 一 & $0.7 \pm 0.1$ & - \\
\hline \multirow[t]{2}{*}{$F_{0.5-1.5 \mathrm{keV}^{d}}$} & $1.7_{-0.5}^{+0.1}$ & $1.7_{-0.7}^{+0.5}$ & $1.7_{-1.4}^{+0.6}$ & $1.7_{-0.9}^{+0.1}$ & $1.8_{-0.5}^{+0.3}$ & $1.1_{-0.4}^{+0.1}$ \\
\hline & $(1.4 \pm 0.2)$ & $\left(1.7_{-1.2}^{+0.1}\right)$ & & & & $(0.8 \pm 0.1)$ \\
\hline \multirow[t]{2}{*}{$F_{1.5-10 \mathrm{keV}^{e}}$} & $2.5_{-1.1}^{+0.2}$ & $2.5_{-1.4}^{+0.3}$ & $2.5_{-2.3}^{+0.5}$ & $2.5_{-0.5}^{+0.3}$ & $2.5_{-0.8}^{+0.3}$ & $3.9_{-1.7}^{+0.4}$ \\
\hline & $(2.5 \pm 0.2)$ & $\left(2.5_{-0.5}^{+0.2}\right)$ & & & & $(3.9 \pm 0.5)$ \\
\hline \multirow[t]{2}{*}{$F_{0.5-10 \mathrm{keV}^{e}}$} & $2.5_{-1.0}^{+0.3}$ & $2.5_{-1.4}^{+0.3}$ & $2.5_{-2.0}^{+0.5}$ & $2.5_{-0.5}^{+0.2}$ & $2.5_{-0.9}^{+0.3}$ & $3.9_{-2.0}^{+0.4}$ \\
\hline & $(2.5 \pm 0.3)$ & $\left(2.5_{-0.5}^{+0.3}\right)$ & & & & $\left(3.9_{-0.5}^{+0.4}\right)$ \\
\hline$\chi_{\text {red }}^{2} /$ d.o.f. & $\begin{array}{c}1.02 / 174 \\
(1.20 / 175)\end{array}$ & $\begin{array}{c}1.02 / 173 \\
(0.99 / 173)\end{array}$ & $0.98 / 173$ & $1.02 / 173$ & $0.98 / 173$ & $\begin{array}{c}1.09 / 183 \\
(1.16 / 184)\end{array}$ \\
\hline
\end{tabular}

Notes. $N_{\mathrm{H}}$ indicates the absorption column density, $\Gamma$ the power law photon index, $E_{\text {cut }}$ the cutoff energy of the CUTOFFPL component, and $R_{\mathrm{BB}}$ the $\mathrm{BB}$ radius. We indicate with $k T$ the temperature of the $\mathrm{BB}$ component, the temperature of the COMPTT seed photons, or the temperature of the optically thin gas, depending on the model. $k T_{\mathrm{e}}$ is the temperature of the Comptonizing electron region in the COMPTT model, and $\tau$ its optical depth. We also report the inferred values of $N_{\mathrm{MKL}}$, which is the normalization of the MKL, $N_{\mathrm{H}_{2}}$, the additional absorption column density predicted by the partial covering model, and $0<f<1$ the covering fraction. Finally, we give the source flux in two different energy bands $(0.5-1.5 \mathrm{keV}, 1.5-10 \mathrm{keV})$, and report also the X-ray flux in the $0.5-10 \mathrm{keV}$ energy band for completeness. ${ }^{(a)}$ in units of $10^{22} \mathrm{~cm}^{-2}$. ${ }^{(b)} \mathrm{For} \mathrm{a}$ distance of $2.7 \mathrm{kpc}{ }^{(c)}$ Absorption column density of the ZPCFABS component. ${ }^{(d)}$ Observed flux in units of $10^{-14} \mathrm{erg} \mathrm{cm}^{-2} \mathrm{~s}^{-1}$. (e) $\mathrm{Observed} \mathrm{flux} \mathrm{in}$ units of $10^{-12} \mathrm{erg} \mathrm{cm}^{-2} \mathrm{~s}^{-1}$.

upper limits at a level of $20 \%, 30 \%$, and $35 \%$ for periods in the range $0.03-20 \mathrm{~s}, 20-50 \mathrm{~s}$, and $0.02-0.03 \mathrm{~s}$, respectively.

\subsection{IGRJ08408-4503}

XMM-Newton observed IGR J08408-4503 on 2007 May 29, with the Epic-PN camera operating in full frame. To identify the high background time intervals we followed the same technique described for XTE J1739-302 (see Sect. 4.1 and Fig. 1). We extracted the Epic-PN lightcurve for the full field of view (FOV) in the 10-12 keV energy band, and set a threshold on the fullFOV count rate in this energy band of $0.45 \mathrm{cts} \mathrm{s}^{-1}$. The total effective exposure time after the good time interval selection for IGR J08408-4503 was $26 \mathrm{ks}$.

From the lightcurve of the observation (Fig. 10), it is apparent that the variability in the quiescent state of this source was rather similar to that of XTE J1739-302. In particular, the lower panel of Fig. 10 shows that the hardness ratio of IGR J084084503 increased with the source count rate. Figure 11 shows the hardness-intensity diagram of IGR J08408-4503, obtained with the same technique described in Sect. 4.1. In this case, the scatter of the points is somehow less evident than in the case of XTE J1739-302 and a linear fit to the data required a slope of $1.9 \pm 0.2$. To investigate the origin of the variability in the hardness ratio of IGR J08408-4503, we extracted three different spectra during the time intervals of the observation in which the source count rate was $>0.2,0.1-0.2$, and $<0.1$ (hereafter spectra A, B, C). A fit to these spectra with a simple absorbed BB or PL model provided unacceptable results $\left(\chi_{\text {red }}^{2} \gtrsim 1.5-5.0\right.$, d.o.f. $=$ 28-44). A CUTOFFPL model with a fixed $E_{\text {cut }}=11 \mathrm{keV}$ (see Sect. 1) provided tighter fits to the three spectra $\left(\chi_{\text {red }}^{2} \sim 1.2-1.6\right.$, d.o.f. $=28-44)$. However, the $\chi_{\text {red }}^{2}$ was still significantly larger than 1 , the value of the absorption column density measured from the spectra B and C was unreasonably low (compatible with zero), and some structures were apparent in the residuals from the fits at energies $<2 \mathrm{keV}$ (see Fig. 12). A CUTOFFPL model with a free to vary $E_{\text {cut }}$ improved only the fit to spectrum $\mathrm{A}\left(\chi_{\mathrm{red}}^{2} /\right.$ d.o.f. $\left.=1.03 / 39\right)$, whereas the results of the fits to the spectra $\mathrm{B}$ and $\mathrm{C}$ were almost unchanged. To fit the $\mathrm{A}, \mathrm{B}$, and $\mathrm{C}$ spectra with the same model, and obtain at the same time reasonable values of the fit parameters (e.g. an $N_{\mathrm{H}}$ at least comparable with the Galactic value in the direction of the source), we assumed that $E_{\text {cut }}=11 \mathrm{keV}$ (as it was found when the source was in outburst, Romano et al. 2009a) and added a second spectral component to account for the residuals below $\sim 2 \mathrm{keV}$. We found that a significant improvement in the fits could be obtained by 
A\&A 519, A6 (2010)

Table 3. The same as Table 2, but for the time-resolved spectra of IGR J08408-4503.

\begin{tabular}{lccccccccc}
\hline \hline Model & \multicolumn{3}{c}{ BB+CUTOFFPL } & \multicolumn{3}{c}{ MKL+CUTOFFPL } & \multicolumn{3}{c}{ BMC } \\
\hline & $<0.1$ & $0.1-0.2$ & $>0.2$ & $<0.1$ & $0.1-0.2$ & $>0.2$ & $<0.1$ & $0.1-0.2$ & $>0.2$ \\
\hline$N_{\mathrm{H}}\left(10^{22} \mathrm{~cm}^{-2}\right)$ & $1.0_{-0.3}^{+0.4}$ & $0.8_{-0.3}^{+0.2}$ & $1.2 \pm 0.3$ & $0.7_{-0.4}^{+0.1}$ & $0.6 \pm 0.2$ & $0.9_{-0.3}^{+0.2}$ & $1.1 \pm 0.3$ & $0.9_{-0.3}^{+0.2}$ & $1.4 \pm 0.3$ \\
$\Gamma$ & $2.1_{-0.3}^{+0.4}$ & $1.7 \pm 0.2$ & $1.3 \pm 0.2$ & $1.7 \pm 0.2$ & $1.6_{-0.2}^{+0.1}$ & $1.1_{-0.2}^{+0.1}$ & $2.6_{-0.2}^{+0.3}$ & $2.1_{-0.1}^{+0.2}$ & $1.7 \pm 0.2$ \\
$k T_{\mathrm{BB}}(\mathrm{keV})$ & $0.08 \pm 0.02$ & $0.08_{-0.01}^{+0.02}$ & $0.08_{-0.02}^{+0.01}$ & - & - & - & - & - & - \\
$R_{\mathrm{BB}}^{a}(\mathrm{~km})$ & $156_{-46}^{+733}$ & $82_{-60}^{+160}$ & $286_{-2.24}^{+795}$ & - & - & - & - & - & - \\
$k T_{\mathrm{MKL}}(\mathrm{keV})$ & - & - & - & $0.22_{-0.04}^{+0.14}$ & $0.21_{-0.04}^{+0.07}$ & $0.20_{-0.04}^{+0.10}$ & - & - & - \\
$N_{\mathrm{MKL}}\left(10^{-3}\right)$ & - & - & - & $1.0_{-0.7}^{+2.0}$ & $1.1_{-1.0}^{+3.6}$ & $7.7_{-6.8}^{+74.0}$ & - & - & - \\
$k T_{\mathrm{BMC}}(\mathrm{keV})$ & - & - & - & - & - & - & $0.07 \pm 0.01$ & $0.08 \pm 0.01$ & $0.07 \pm 0.01$ \\
$\log (A)$ & - & - & - & - & - & - & $-2.8_{-0.2}^{+0.5}$ & $-2.1_{-0.3}^{+0.4}$ & $-2.9_{-0.5}^{+0.7}$ \\
$N_{\mathrm{BMC}}\left(10^{-3}\right)$ & - & - & - & - & - & - & $4.3_{-3.7}^{+22.3}$ & $1.1_{-1.1}^{+2.5}$ & $16.4_{-14.4}^{+75.5}$ \\
$F_{0.5-1.5 \mathrm{keV}}^{b}$ & $0.84_{-0.76}^{+0.05}$ & $1.4_{-1.0}^{+0.1}$ & $3.0_{-2.1}^{+0.2}$ & $0.84_{-0.64}^{+0.12}$ & $1.4_{-0.9}^{+0.2}$ & $3.0_{-1.8}^{+0.1}$ & $0.83_{-0.83}^{+0.12}$ & $1.4_{-1.4}^{+0.3}$ & $2.9_{-2.0}^{+0.2}$ \\
$F_{1.5-10 \mathrm{keV}}^{b}$ & $2.2_{-1.3}^{+0.4}$ & $6.2_{-2.8}^{+1.1}$ & $38.1_{-12.0}^{+6.0}$ & $2.3_{-0.8}^{+0.5}$ & $6.3_{-2.1}^{+1.0}$ & $38.5_{-10.1}^{+5.5}$ & $2.2_{-1.3}^{+0.4}$ & $6.2_{-6.2}^{+1.4}$ & $38.6_{-32.6}^{+1.1}$ \\
$F_{0.5-10 \mathrm{keV}}^{b}$ & $3.0_{-1.9}^{+0.4}$ & $7.6_{-3.3}^{+1.0}$ & $41.1_{-17.0}^{+5.0}$ & $3.2_{-1.5}^{+0.4}$ & $7.7_{-3.2}^{+1.2}$ & $41.5_{-12.0}^{+5.0}$ & $2.9_{-2.7}^{+0.4}$ & $7.7_{-7.7}^{+1.3}$ & $41.5_{-29.8}^{+1.1}$ \\
$\chi_{\text {red }}^{2} /$ d.o.f. & $0.8 / 26$ & $1.2 / 42$ & $0.7 / 38$ & $0.8 / 26$ & $1.2 / 42$ & $0.8 / 38$ & $0.8 / 26$ & $1.2 / 42$ & $0.7 / 38$ \\
EXP. (ks) & 13 & 9 & 2 & 13 & 9 & 2 & 13 & 9 & 2 \\
\hline
\end{tabular}

Notes. In all cases, the cutoff energy is fixed at $11 \mathrm{keV}$ (see Sect. 4.2). For the BMC model, we reported below the value $\Gamma=\alpha+1$, where $\alpha$ is the Comptonization efficiency of the model, the value of the "illumination parameter" $\log (A)$, and the normalization $N_{\mathrm{BMC}}{ }^{\left({ }^{(a)}\right.}$ For a distance of $3 \mathrm{kpc}$. (b) Observed flux in unit of $10^{-13} \mathrm{erg} \mathrm{cm}^{-2} \mathrm{~s}^{-1}$. (c) Exposure time.

IGRJ08408-4503 (Epic-PN)

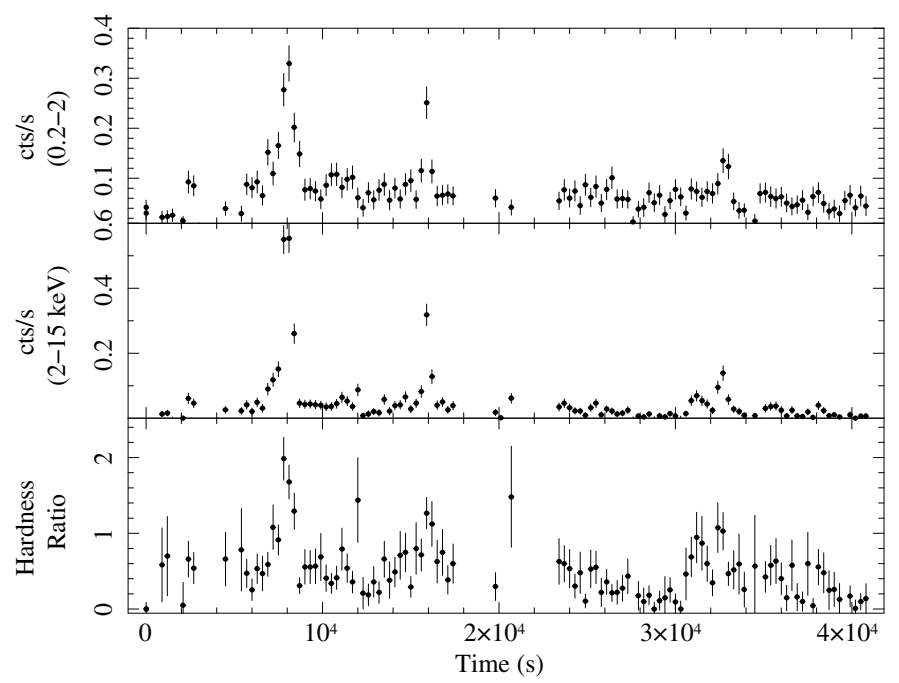

Fig. 10. XMM-Newton Epic-PN background-subtracted lightcurve of IGR J08408-4503 during the observation of 2007 May 29. The upper and middle panel shows the source lightcurve in the $0.2-2 \mathrm{keV}$ and $2-$ $15 \mathrm{keV}$ energy bands, respectively. The binning time is $300 \mathrm{~s}$. The ratio of the source count rate in the two bands versus time is shown in the lower panel.

adding a MKL or a BB component. A single BMC model also provided a reasonable fit to the data. The results of all these fits are reported in Table 3 . The significance of the improvement obtained by using these two-component models instead of a simple CUTOFFPL is 4,3 , and $4.1 \sigma$, respectively, for the spectra A, B, and $\mathrm{C}$.

By looking at the results of the fits in Table 3, we conclude that the increase in the source hardness ratio with the count rate can be most likely ascribed to a variation in the photon index, $\Gamma$, rather than a change in the absorption column density or in

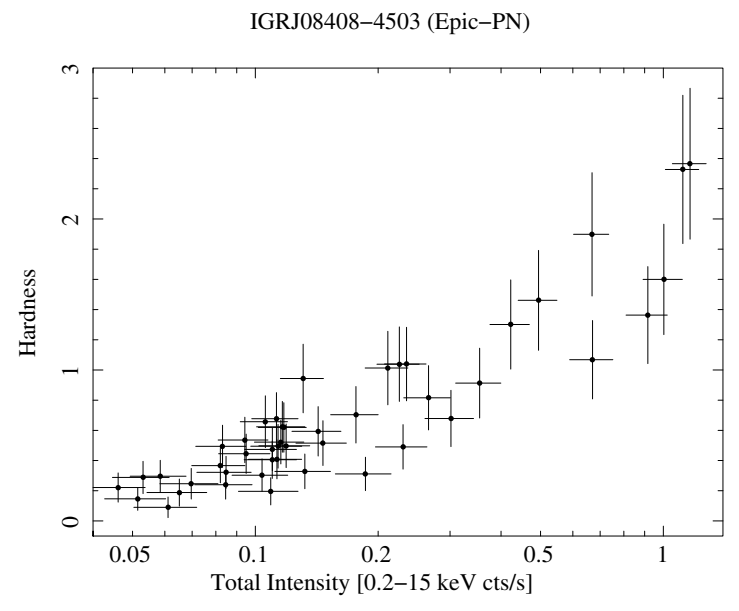

Fig. 11. Hardness-intensity diagram for IGRJ08408-4503. The diagram was created by using the $0.2-2 \mathrm{keV}$ and $2-15 \mathrm{keV}$ lightcurves of Fig. 10, but rebinned so as to achieve a $S / N \gtrsim 5.5$ in all the hardness ratio values. The hardness is defined as the ratio of the count rate in the hard $(2-15 \mathrm{keV})$ to soft $(0.2-2 \mathrm{keV})$ energy band.

the properties of the soft component. Indeed, the value of $N_{\mathrm{H}}$, as well as the temperature and normalization of the BB and the MKL components, are compatible with being constant (to within the errors) in the three spectra. That most of the changes in the Xray flux of the source occurred in the $1.5-10 \mathrm{keV}$ energy band, where the contribution of the CUTOFFPL component is much greater than that of the soft component (see Fig. 9), added support fot the above conclusion.

To further constrain the soft component more tightly, we also extracted the source spectrum by using the total available exposure time of the XMM-Newton observation. A fit to this spectrum with a simple BB or a PL model did not provide an acceptable result $\left(\chi_{\text {red }}^{2} /\right.$ d.o.f. $=4.9 / 101,1.8 / 101$, respectively for the BB and the PL model). A CUTOFFPL model 


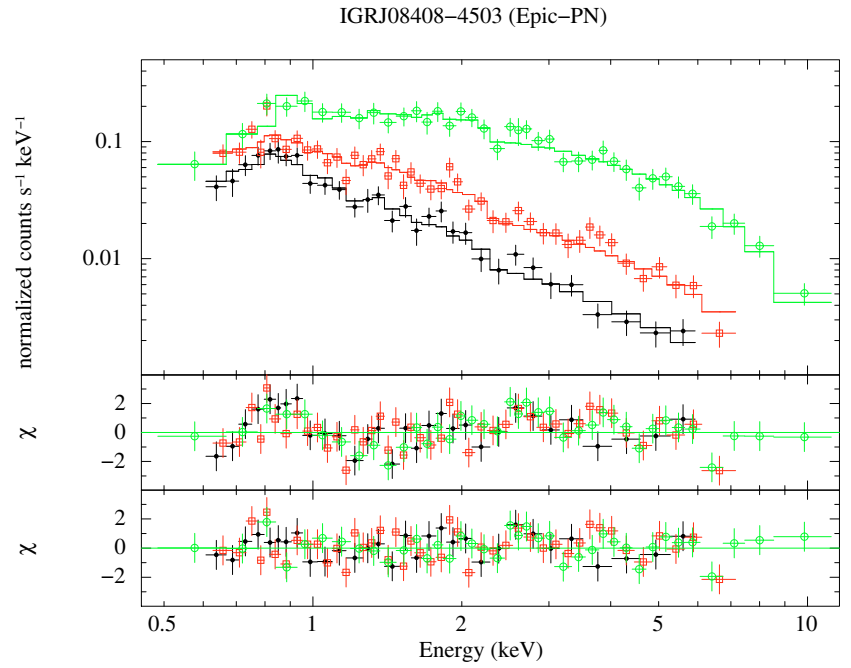

Fig. 12. The same as Figs. 5 and 6 but for the observation of IGR J08408-4503. Here the open circles, open squares, and filled circles refer to the spectrum accumulated during the time intervals of the observation in which the Epic-PN $0.2-15 \mathrm{keV}$ source rate was $>0.2$, $0.1-0.2,<0.1$ counts/s respectively. The model used for the best fits comprises a MKL plus a CUTOFFPL component ( $E_{\text {cut }}$ fixed at $11 \mathrm{keV}$ ). The residuals from these fits are shown in the lower panel. The middle panel shows the residuals of the fits obtained by using a simple absorbed CUTOFFPL model (cutoff energy fixed at $11 \mathrm{keV}$ ). (See the electronic edition of the paper for a color version of this figure.)

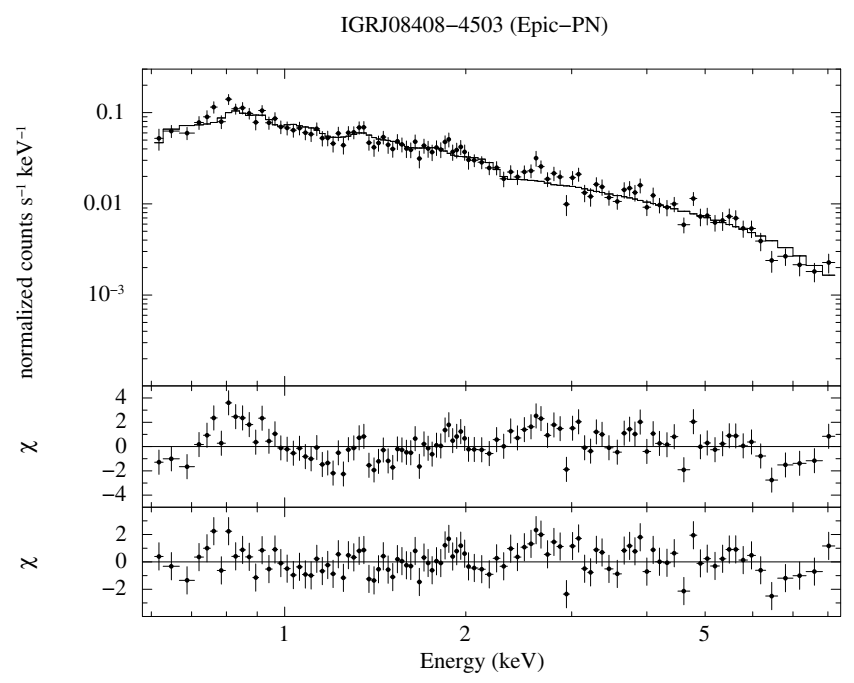

Fig. 13. The XMM-Newton spectrum of IGR J08408-4503 extracted by using the total exposure time of the observation carried out on 2007 May 29. The model used for the fit is an absorbed CUTOFFPL plus a MKL (the cutoff energy is fixed at $11 \mathrm{keV}$ ). The residuals from the fit are shown in the bottom panel. The middle panel shows the residuals of the fit obtained by using only an absorbed CUTOFFPL model (cutoff energy is fixed at $11 \mathrm{keV}$ ).

only marginally improved the fit $\left(\chi_{\text {red }}^{2} /\right.$ d.o.f. $\left.=1.5 / 100\right)$ and some structures remained present in the residuals (see Fig. 13). To obtain an acceptable fit to the data, we thus used the same spectral models that we adopted for the rate-resolved analysis. All these models provided an equivalently good fit to the data. The results of these fits are given in Table 4 and discussed in detail in the following section. We note that when fitting the total spectrum of IGR J08408-4503 we discarded data in the energy range $0.4-0.6 \mathrm{keV}$, as we noted that in this energy range
Table 4. The same as Table 3, but for the spectrum of IGR J08408-4503 obtained by using the total available exposure time of the XMM-Newton observation.

\begin{tabular}{lccc}
\hline \hline Model & BB+CUTOFFPL & MKL+CUTOFFPL & BMC \\
\hline$N_{\mathrm{H}}\left(10^{22} \mathrm{~cm}^{-2}\right)$ & $0.9_{-0.1}^{+0.2}$ & $0.7_{-0.2}^{+0.1}$ & $1.0_{-0.2}^{+0.1}$ \\
$\Gamma$ & $1.6 \pm 0.1$ & $1.4 \pm 0.1$ & $2.0 \pm 0.1$ \\
$k T_{\mathrm{BB}}(\mathrm{keV})$ & $0.082_{-0.004}^{+0.007}$ & - & - \\
$R_{\mathrm{BB}}{ }^{a}(\mathrm{~km})$ & $100_{-47}^{+126}$ & - & - \\
$k T_{\mathrm{MKL}}(\mathrm{keV})$ & - & $0.22_{-0.02}^{+0.04}$ & - \\
$N_{\mathrm{MKL}}\left(10^{-3}\right)$ & - & $1.3_{-0.8}^{+1.1}$ & - \\
$k T_{\mathrm{BMC}}(\mathrm{keV})$ & - & - & $0.075_{-0.006}^{+0.007}$ \\
$\log (A)$ & - & - & $-2.5_{-0.2}^{+0.3}$ \\
$N_{\mathrm{BMC}}\left(10^{-3}\right)$ & - & - & $2.0_{-1.3}^{+2.8}$ \\
$F_{0.5-1.5 \mathrm{keV}^{b}}$ & $1.2_{-0.8}^{+0.1}$ & $1.2_{-0.7}^{+0.1}$ & $1.2_{-0.8}^{+0.2}$ \\
$F_{1.5-10 \mathrm{keV}^{b}}$ & $6.3_{-1.4}^{+0.7}$ & $6.3_{-1.0}^{+0.7}$ & $6.4_{-3.7}^{+0.9}$ \\
$F_{0.5-10 \mathrm{keV}^{b}}$ & $7.5_{-2.1}^{+0.6}$ & $7.5_{-1.5}^{+0.8}$ & $7.5_{-4.0}^{+1.1}$ \\
$\chi_{\text {red }}^{2} / \mathrm{d} .0 . f$. & $0.99 / 99$ & $1.05 / 99$ & $1.02 / 99$ \\
$\mathrm{EXP}(\mathrm{ks})^{c}$ & 26 & 26 & 26 \\
\hline
\end{tabular}

Notes. ${ }^{(a)}$ For a distance of $3 \mathrm{kpc}$. ${ }^{(b)}$ Observed flux in units of $10^{-13} \mathrm{erg} \mathrm{cm}^{-2} \mathrm{~s}^{-1}$. ${ }^{(c)}$ Exposure time.

the background was rather high. Including these points does not affect the best-fit values of the model parameters, but indicates that the fit with a CUTOFFPL+BB model is slightly preferable $\left(\chi_{\text {red }}^{2} /\right.$ d.o.f. $\left.=1.0 / 104\right)$ than the CUTOFFPL+MKL model $\left(\chi_{\text {red }}^{2} /\right.$ d.o.f. $\left.=1.1 / 104\right)$. Further XMM-Newton observations of this source are probably needed to resolve this issue. In Fig. 13, we show the spectrum of IGR J08408-4503 accumulated over the entire exposure of the observation and fitted with the MKL+CUTOFFPL model. In this figure, we also show for comparison the residuals obtained by fitting the same spectrum with a simple absorbed CUTOFFPL model $\left(E_{\text {cut }}=11 \mathrm{keV}\right.$, fixed). The unfolded spectrum of IGR J08408-4503 is shown in Fig. 14.

We searched for pulsations in the power spectra of the XMMNewton observation of IGRJ08408-4503 by using the same technique described in Sect. 4.1. No significant (above $3 \sigma$ level) signal was detected in these data. We determined an upper limit to the pulsed fraction of $25 \%, 30 \%$, and $40 \%$ for periods in the range $0.3-50 \mathrm{~s}, 50-100 \mathrm{~s}$, and $0.15-0.3 \mathrm{~s}$, respectively ( $3 \sigma \mathrm{c} .1$. $)$.

\section{Discussion}

We have presented the first deep pointed observations of the two prototypical SFXTs, XTE J1739-302 and IGR J08408-4503 in quiescence.

The two sources exhibited a very complex timing and spectral variability, and we discuss them separately below. Here we also carry out a comparison between their quiescent and outburst emission.

\subsection{XTE J1739-302}

The two XMM-Newton observations analyzed in Sect. 4.1 show that the quiescent emission of XTE J1739-302 is characterized by a number of low-intensity flares, taking place sporadically from a lower persistent emission level. The typical duration 
IGRJ08408-4503 (Epic-PN)

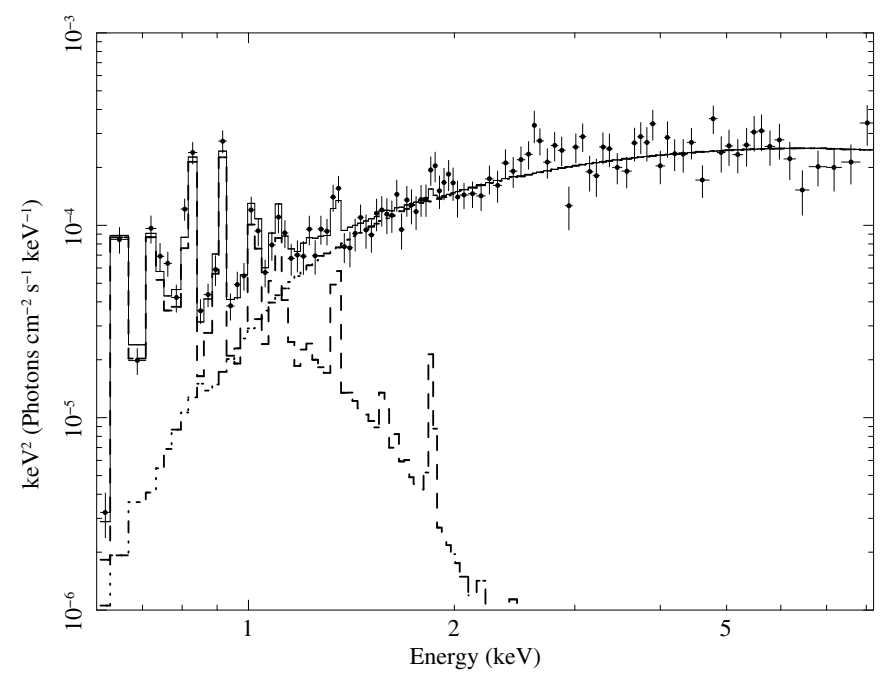

Fig. 14. The unfolded spectrum of the XMM-Newton observation of IGR J08408-4503. The best-fit model represented here is obtained by using an absorbed CUTOFFPL (cutoff energy fixed at $11 \mathrm{keV}$ ) plus a MKL component.

of these flares is a few thousands of seconds, and their Xray flux is a factor of $\sim 10-30$ higher than the persistent quiescent flux. During the time intervals when the source was at its lowest level of emission, we measured an X-ray flux of $4.7 \times 10^{-13} \mathrm{erg} \mathrm{cm}^{-2} \mathrm{~s}^{-1}(0.5-10 \mathrm{keV})$. This corresponds to a luminosity of $4.1 \times 10^{32} \mathrm{erg} \mathrm{s}^{-1}$ at $2.7 \mathrm{kpc}$, and is among the lowest values of X-ray luminosity measured from XTE J1739302 (a factor of $\sim 2$ lower than the $3 \sigma$ upper limit reported by Sakano et al. (2002), and comparable with the lowest luminosity reported by Romano et al. 2009b). The total dynamic range of the X-ray luminosity of XTE J1739-302 between outburst and quiescence is thus $\gtrsim 10^{4}$ (see Sect. 1 ).

The hardness intensity diagrams and rate resolved analysis carried out in Sect. 4 showed that the variations in the X-ray flux measured during the XMM-Newton observations were accompanied by a change in the spectral properties of the source, the source hardness ratio increasing significantly with the count rate. A fit to the rate-resolved spectra with a CUTOFFPL model ( $E_{\text {cut }}=13 \mathrm{keV}$ Sidoli et al. 2009a) implies that this behavior originates from a change in the CUTOFFPL photon index, $\Gamma$, rather than in a variation of the absorption column density.

These results indicate that the timing and spectral variability of XTE J1739-302 during the quiescence is qualitatively similar to that observed during the outbursts (see Sect. 1). A change in the PL photon index with the X-ray flux of XTE J1739-302 was first noticed by Smith et al. (1998). These authors analyzed several different flares caught by RXTE, INTEGRAL and ASCA, and showed that the photon index of the hard (2-10 keV) Xray emission from XTEJ1739-302 changed from 0.8, when the source X-ray flux was $2.4 \times 10^{-9} \mathrm{erg} \mathrm{s}^{-1}(2-10 \mathrm{keV})$, to 2.0 , when the $\mathrm{X}$-ray flux was $1.6 \times 10^{-9} \mathrm{erg} \mathrm{s}^{-1}(2-10 \mathrm{keV})$. However, these flares were also characterized by a significant change in the absorption column density (from 3 to $37 \times 10^{22} \mathrm{~cm}^{-2}$ ). Similar values of the PL photon index and absorption column density were reported for the outbursts observed with Swift from this source (see Sect. 1). In the long-term monitoring of XTE J1739-302 carried out with Swift, Romano et al. (2009b) identified three different states in the source X-ray flux (Sect. 1), and showed that each of these states could be characterized by a different PL photon index (in the range 0.5-1.6) and a different absorption column density (from 0.3 to $3.6 \times 10^{22} \mathrm{~cm}^{-2}$ ). Considering all of these results, it seems unlikely that there exists a single monotonic variation in both the absorption column density and PL photon index with the source intensity across its quiescent and outburst state.

When the X-ray spectrum of XTE J1739-302 from the entire XMM-Newton observation is considered, the interpretation of the emission from this source becomes even more complicated. In particular, this spectrum cannot be fit successfully by using a simple absorbed PL or a BB model. We indeed showed in Sect. 4.1 that these models provided only a poor fit to the data, and a more refined model was required. Because of the relatively low X-ray flux of the source, different models could equivalently describe the data. From a statistical point of view, the CUTOFFPL with a variable cutoff energy would be preferable, as it provided a very good fit to the data of both OBS1 and OBS2 and requires a lower number of free parameters with respect to the others models reported in Table 2. However, this model would require a cutoff energy much lower $(\sim 4 \mathrm{keV})$ than the value determined when the source was in outburst $(\sim 13 \mathrm{keV})$. This low value for the cutoff energy might not be unlikely for an X-ray pulsar (see e.g., the cases of X-Persei and RX J0146.9+61214 , Haberl et al. (1998); Di Salvo et al. (1998), and Coburn et al. (2002) for a review). However, we suggested that, given our relatively poor knowledge of the quiescent emission of XTE J1739-302 and of SFXTs in general, the applicability of other spectral models might be worth exploring.

Besides the CUTOFFPL, the quiescent spectrum of XTE J1739-302 might require an additional spectral component at the lower energies $(<2 \mathrm{keV})$. In Sect. 4.1, we showed that a fit to the spectra of OBS1 and OBS2 with a CUTOFFPL model and a fixed $E_{\text {cut }}=13 \mathrm{keV}$ would leave some evident structures in the residuals from the fit and the addition of a BB, or a MKL component can significantly improve the results. Even if these models require one more free parameter than the CUTOFFPL with a variable $E_{\text {cut }}$, the results we obtained would then be in agreement with those found in the case of IGR J08408-4503. The spectrum of this source could, indeed, not be reproduced using a simple CUTOFFPL model (see below).

A soft spectral component below $2 \mathrm{keV}$ might be expected in the spectra of the SFXT sources, as this component is very common in binaries hosting a NS accreting mass from a massive companion. Hickox et al. (2004) showed that the detectability of this component depends mainly on the amount of absorption in the direction of the sources. According to their results, in the most luminous objects $\left(L_{X} \simeq 10^{38} \mathrm{erg} \mathrm{s}^{-1}\right)$ the soft component is produced by reprocessing hard X-rays from the NS by some optically thick material (e.g., an accretion disk), whereas for sources with lower luminosities $\left(<10^{36} \mathrm{erg} \mathrm{s}^{-1}\right)$ the most likely origin of the soft component is the emission from either a photoionized or collisionally heated diffuse gas in the binary system or from the NS surface. In the case of XTE J1739-302, we found that $\mathrm{BB}$ emission from a relatively cold $(\sim 0.1 \mathrm{keV})$ and large ( $\sim 100 \mathrm{~km}$ equivalent radius) region or from a much hotter $(\gtrsim 1 \mathrm{keV})$ and less extended $(<100 \mathrm{~m})$ spot, or, alternatively, emission from an optically thin gas (MKL) provided equally good fits to the data. While the discussion above and the rapid variability observed in the SFXT would argue against the presence of an accretion disk in these sources (see also, Bozzo et al. 2008), we also consider that emission from a small and hot spot

\footnotetext{
4 Note that these authors used an absorbed power-law with exponential high-energy cutoff (HIGHECUT ${ }^{*}$ PL in XSPEC) to fit their data.
} 
on the NS surface is inconsistent with models of accretion onto magnetic NS. The accretion flow in SFXT is, indeed, thought to be quasi-spherical, and expected to penetrate the NS magnetosphere mainly by means of the Rayleigh-Taylor and the KelvinHelmholtz instability (see e.g., Bozzo et al. 2008, and references therein). In these circumstances, the size of the hot-spot over which the accretion takes place is expected to be inversely proportional to the X-ray luminosity and might cover a substantial fraction of the NS surface for $L_{\mathrm{X}} \lesssim 10^{35} \mathrm{erg} \mathrm{s}^{-1}$ (see White et al. 1983, and references therein).

Taking these results into account, emission from an optically thin and diffuse gas around the NS seems to be a more reasonable explanation of the soft spectral component of XTE J1739302. According to this interpretation, the emitting region can be estimated from the normalization of the MKL component (see Table 2) using the relation (see e.g., Zurita Heras \& Walter 2009 , and references therein): $R_{\mathrm{em}}=\sqrt[3]{3 N_{\mathrm{MKL}} / 10^{-14}\left(D / n_{\mathrm{H}}\right)^{2}} \sim$ $10^{13} a_{13}^{2 / 3} \mathrm{~cm}$, where $N_{\mathrm{MKL}}$ is the normalization of the MKL component, $\mathrm{D}$ is the source distance, $n_{\mathrm{H}} \sim N_{\mathrm{H}} / a, a$ is the binary separation, and $a_{13}=a / 10^{13} \mathrm{~cm}$. Therefore, the radius $R_{\mathrm{em}}$ of the emitting region is compatible with the binary separation for a wide range of values of orbital periods similar to those measured in other SFXTs. We note also that the properties of this MKL component would be rather similar to those derived from the spectrum of the SFXT AX J1845.0-0433 (Zurita Heras \& Walter 2009).

Another possibility that we investigated in Sect. 4.1 is the applicability of a model comprising a power law component and a partial covering to the X-ray spectrum of XTE J1739-302. We concluded that this model can also provide a reasonably good fit to the data. A similar model was proposed to interpret the quiescent XMM-Newton spectrum of the SFXT IGR J16207-5129 (Tomsick et al. 2009) and might provide support for clumpy wind in these sources. In this case, one would expect part of the radiation from the NS to escape absorption by the clumps local to the source and be affected only by interstellar absorption (see, e.g. Walter \& Zurita Heras 2007). Finally, we showed that the COMPTT model also provided a reasonable fit to the XMM-Newton spectra of XTE J1739-302, and should thus be considered a valid alternative to the other models discussed above. We note, however, that neither the partial covering nor the COMPTT model could give an acceptable fit to the spectrum of IGR J08408-4503 (see also below). Given the similarities between the two sources, a spectral model that provides acceptable results in both cases should probably be favored (e.g., the CUTOFFPL+MKL model).

\subsection{IGR J08408-4503}

The XMM-Newton observation of IGR J08408-4503, detected a very similar behavior to that discussed above for XTE J1739302 . In the light curve of IGR J08408-4503, a number of relatively small flares were observed to take place sporadically on a timescale of few thousands of seconds and were characterized by an X-ray flux $\sim 10-15$ times higher than the underlying fainter persistent emission. The lowest X-ray flux that we measured from this source was $\sim 3 \times 10^{-13} \mathrm{erg} \mathrm{cm}^{-2} \mathrm{~s}^{-1}(0.5-10 \mathrm{keV})$ and corresponds to a luminosity of $3.3 \times 10^{32} \mathrm{erg} \mathrm{s}^{-1}$ (at a distance of $3 \mathrm{kpc}$ ), comparable to the value reported by Kennea \& Campana (2006). The total dynamic range in the X-ray luminosity of IGR J08408-4503 between outburst and quiescence is thus $\gtrsim 10^{4}$ (see also Sect. 1).
As for XTE J1739-302, the hardness intensity diagrams and the rate resolved analysis carried out in Sect. 4 showed that the variations in the X-ray flux measured during the XMM-Newton observation were also accompanied by a significant change in the spectral properties of the source. In contrast to the case of XTEJ1739-302, the rate-resolved spectra of IGR J084084503 could not be fit by using a simple CUTOFFPL model. We showed that an acceptable fit to the data could, instead, be obtained by introducing an additional relatively cold $(k T \sim$ $0.08 \mathrm{keV})$ and extended $(\gtrsim 100 \mathrm{~km}) \mathrm{BB}$ component, or a MKL model (see Tables 3 and 4). We note that the parameters measured for the MKL component are rather similar to those derived in the case of XTE J1739-302. An equivalently good fit was provided by the BMC model. This model has the same number of free parameters as the CUTOFFPL+BB (see Table 3), and would predict similar properties for the temperature and the size in which the soft photons are produced (the normalization of the BMC model is defined as the ratio of the source luminosity to the square of the distance in units of $10 \mathrm{kpc}$, see e.g. Sidoli et al. 2009b, and references therein). Similar values of the fit parameters were also obtained from the analysis of the spectrum of IGR J08408-4503 extracted by using the total available exposure time of the XMM-Newton observation (see Sect. 4.2 and Table 4).

According to the discussion in Sect. 5.1, a BB emission with these properties seems unlikely in the case of IGR J084084503 and thus we suggest that the CUTOFFPL+MKL model can provide a more reasonable description of the data. We note that in the XMM-Newton spectrum of the supergiant HMXB IGR J16320-4751 a similar soft component was found that could be fit with a BB of $0.07 \mathrm{keV}$ but was attributed to a cloud surrounding the NS (Rodriguez et al. 2006). Following the CUTOFFPL+MKL interpretation, the rate resolved analysis carried out for the observation of IGR J08408-4503 would indicate that the properties of the MKL component do not change significantly with the source count rate and the increase in the hardness ratio observed in Figs. 11 and 10 is most likely due to a change in the CUTOFFPL photon index. Furthermore, no significant variations in the absorption column density were revealed in the different rate-resolved spectra. This is similar to the results discussed above for XTE J1739-302.

As for XTE J1739-302, a comparison between the results of the present XMM-Newton observation and the observations carried out in the same energy band $(0.5-10 \mathrm{keV})$ when this source was in outburst does not indicate a clear correlation between the power law photon index, the absorption column density, and the source X-ray flux (see Sect. 1). It is interesting that the soft component in this source detected by the XMM-Newton observation appears to have a different origin from that detected by Sidoli et al. (2009b) when IGR J08408-4503 was in outburst (see Sect. 2.2). On that occasion, the soft component appeared to be caused by thermal emission from a hot-spot on the NS surface. We note that, even if the soft component observed by XMMNewton is interpreted in terms of a BB emission, the emitting region derived from the fit is considerably larger than the NS radius and it is thus unlikely that it is produced on the NS surface.

Finally, for both XTE J1739-302 and IGR J08408-4503, we investigated whether the harder spectral component detected in these sources might be produced by the X-ray emission from the NS supergiant companion. The time-averaged X-ray luminosity that we measured from these sources in quiescence matches quite well the luminosity expected from an isolated OB supergiant or from colliding winds in a binary containing OB supergiant stars (see e.g., Gudel \& Nazel 2009, for a review). 
However, this interpretation appears to be contrived for the following reasons. The X-ray spectrum of isolated or colliding wind binaries with $\mathrm{OB}$ supergiant stars is usually described well by a model comprising one or more thermal components (MKL in XSPEC, see e.g., Gudel \& Nazel 2009). The softer MKL component has a typical temperature of $\sim 0.2-0.7 \mathrm{keV}$, and is thus similar to those we detected in IGR J08408-4503 and XTE J1739-302. This component is thought to be generated by the shocks within the stellar wind. The hotter MKL component, extending up to several $\mathrm{keV}$, can have a temperature as high as $\sim 1-3 \mathrm{keV}$ and is characterized by a number of very prominent emission lines (see also Raassen et al. 2008). This hard component is usually interpreted in terms of magnetically confined wind shocks, highly compressed wind shocks, or inverse Compton scattering of photospheric UV photons by relativistic particles accelerated within the shocks (Albacete Colombo et al. 2007). Possible detections of a non-thermal X-ray emission from OB supergiant stars were reported in only two cases, but they still lack confirmation (Gudel \& Nazel 2009).

The X-ray spectra of XTE J1739-302 and IGR J08408-4503 were reproduced well using a CUTOFFPL model, and no prominent emission lines were detected. The values of the photon index, $\Gamma$, derived from XMM-Newton are also comparable to those obtained previously when the sources were in outburst, thus suggesting that a common mechanism produces their harder X-ray component. Furthermore, the relatively rapid X-ray variability (of period few thousands seconds) observed in the lightcurves of IGR J08408-4503 and XTE J1739-302 is not reminiscent of the typical X-ray variability of the OB stars, which, when present, takes place on longer timescales (tens of ks, see e.g., Albacete Colombo et al. 2007).

We conclude that the harder X-ray emission from XTE J1739-302 and IGR J08408-4503 is most likely due to residual accretion taking place onto the NS at a much lower rate than during outburst.

\section{Conclusions}

The three XMM-Newton observations that we have analyzed in the present work, indicate that the quiescent spectra of the two prototypical SFXT XTE J1739-302 and IGR J08408-4503 are characterized by two different spectral components, one dominating the spectrum at the softer energies $(\lesssim 2 \mathrm{keV})$ and the other one being more prominent above $2 \mathrm{keV}$.

The properties of the soft component $(\$ 2 \mathrm{keV})$ could be reasonably well constrained in the case IGR J08408-4503, where the absorption column density was relatively low $\left(<10^{22} \mathrm{~cm}^{-2}\right)$, whereas in the case of XTE J1739-302 the detection of this component is less significant. However, the similarity in the timing and spectral behavior observed in the quiescent state of the two sources argues in favor of adopting the same spectral model for both of them. We suggested that the model comprising a CUTOFFPL component at the higher energies plus a MKL component would provide a reasonable description of the data and a plausible physical explanation of the properties observed in the two sources (Sect. 5). According to this interpretation, the MKL component would represent the contribution to the total X-ray emission of the shocks in the wind of the supergiant companion. The results of the fits with this model to the data of the three observations inferred a temperature of the MKL component and an emitting region comparable with the values found also in the case of the SFXT AX J1845.0-0433.

Similar soft spectral components have been detected in many other HMXBs and SGXBs. In a few cases, the detection of a number of prominent emission lines in the high resolution $\mathrm{X}$-ray spectra of these sources carried out with the gratings onboard Chandra and the RGS onboard XMM-Newton (see e.g., Watanabe et al. 2006) have convincingly demonstrated that these components are produced by the stellar wind around the NS, and proved to be a powerful diagnostic to probe the structure and composition of the stellar wind in these systems. The statistics of the present XMM-Newton observations is far too low to permit a similar in-depth study of the stellar wind in the case of XTE J1739-302 and IGR J08408-4503. Furthermore, because of the relatively low luminosity and the high absorption that characterize the emission of these sources in quiescence, observations at higher spectroscopic resolution are probably too challenging for the present generation of X-ray satellites, and the improved sensitivity of the X-ray spectrometers planned for future X-ray missions (e.g. IXO) is probably required to firmly establish the presence of a soft spectral component in the quiescent emission of the SFXT sources and shed light on its nature.

If the harder X-ray emission $(2-10 \mathrm{keV})$ detected from the XMM-Newton observations of XTE J1739-302 and IGR J084084503 was really produced by residual accretion as we argued in the previous section, then the accretion process in these sources would take place over more than 4 orders of magnitude of $\mathrm{X}$ ray luminosity ${ }^{5}$. This is similar to the results reported for the SFXT IGR J17544-2619 (Rampy et al. 2009) and, possibly, for the SFXT SAX J1818.6-1703 (in the latter case the origin of the lowest quiescent emission remains unclear, Bozzo et al. 2009). In the case of IGR J17544-2619, Rampy et al. (2009) ascribed the high dynamic range in the X-ray luminosity to the accretion of clumps from the wind of the supergiant star with a high density contrast with respect to the surrounding homogeneous wind. However, it was also suggested that a similar variability might result from the transition across different accretion regimes onto the NS (Bozzo et al. 2008). We note that a similar scenario can be envisaged for interpreting the variations in the X-ray flux observed during the multiple small flares detected in the present observations. Even though they took place at a much lower luminosity level than the brightest outbursts (a factor of $\sim 10^{3}-10^{4}$ ), our analysis showed that all these events shared a number of similar timing and spectral properties. In particular, the timescales on which the smaller flares develop is comparable with the decay timescale of the source luminosity during the outbursts (see Sect. 1), and the spectral photon indices and absorption column densities measured from the XMM-Newton observations are also in qualitative agreement with those reported previously when the sources were observed at a much higher X-ray luminosity level (see Sect. 2). It is thus most likely that the transitions between the lower quiescent states and the small flares detected by XMMNewton from XTE J1739-302 and IGR J08408-4503 might have been triggered by the same mechanism that sometimes gives rise to the brightest outbursts (i.e., the accretion of clumps from the stellar wind and/or the transition between different accretion regimes of the NS, see Sect. 1).

In contrast to the case for the SFXT IGR J18483-0311, we did not detect any pulsation in the quiescent emission of either XTE J1739-302 or IGR J08408-4503, and provided in Sects. 4.1 and 4.2 the corresponding upper limits to the spin periods and pulsed fractions we were able to infer from the present data.

Deep pointed observations of SFXTs in quiescence are still required in order to understand the origin of the peculiar X-ray

\footnotetext{
5 That we did not find any evidence for X-ray eclipses in the three $X M M-N e w t o n$ observations that we analyzed (this is unlike the case of IGR J16479-4514, see Sect. 1) is also consistent with this interpretation.
} 
variability of these sources and distinguish between different models proposed to interpret their behavior.

Acknowledgements. E.B. thanks N. Schartel and the XMM-Newton staff for the rapid schedule of the XMM-Newton observation of XTE J1739-302 after the outburst occurred on 2009 March 10, and R. Farinelli for helpful discussions. We thank the anonymous referee for useful comments.

\section{References}

Albacete Colombo, J. F., Flaccomio, E., Micela, G., Sciortino, S., \& Damiani, F. 2007, A\&A, 464, 211

Bamba, A., Yokogawa, J., Ueno, M., Koyama, K., \& Yamauchi, S. 2001, PASJ, 53,1179

Barthelmy, S. D., Romano, P., Burrows, D. N., et al. 2009, Astr. Tel., 2178

Bird, A. J., Bazzano, A., Hill, A. B., et al. 2009, MNRAS, 393, 11

Blay, P., Martínez-Núnez, S., Negueruela, I., et al. 2008, A\&A, 489, 669

Bozzo, E., Falanga M., \& Stella L. 2008, ApJ, 683, 1031

Bozzo, E., Giunta, A., Stella, L., et al. 2009, A\&A, 502, 21

Clark, D. J., Hill, A. B., Bird, A. J., et al. 2009, MNRAS, 399, 113

Coburn, W., Heindl, W. A., Rothschild, R. E., et al. 2002, ApJ, 580, 394

di Salvo, T., Burderi, L., Robba, N. R., \& Guainazzi, M. 1998, ApJ, 509, 897

Dickey, J. M., \& Lockman, F. J. 1990, ARA\&A, 28, 215

Giunta, A., Bozzo, E., Bernardini, F., et al. 2009, MNRAS, 399, 744

Götz, D., Falanga, M., Senziani, F., et al. 2007, ApJ, 655, L101

Grebenev, S. A., \& Sunyaev, R. A. 2007, AstL, 33, 149

Gudel, M., \& Naze, Y. 2009, A\&ARv, 17, 309

Haberl, F., Angelini, L., \& Motch, C. 1998, A\&A, 335, 587

Hickox, R. C., Narayan, R., \& Kallman, T. R. 2004, ApJ, 614, 881

in 't Zand 2005, A\&A, 441, L1

Israel, G. L., \& Stella, L. 1996, ApJ, 468, 369

Jain, C., Biswajit, P., \& Anjan, D. 2009, MNRAS, 397, 11

Kennea, J. A., \& Campana, S. 2006, Astr. Tel., 818

Leyder, J.-C., Walter, R., Lazos, M., Masetti, N., \& Produit, N. 2007, A\&A, 465, L35
Lutovinov, A., Revnivtsev, M., Gilfanov, M., et al. 2005, A\&A, 444, 821 Masetti, N., Pretorius, M. L., Palazzi, E., et al. 2006, A\&A, 449, 1139 Negueruela, I., Torrejon, J. M., Reig, P., Ribo, M., \& Smith, D. M. 2008, AIP Conf. Proc., 1010, 252

Oskinova, L. M., Harmann, W. R., \& Feldmeier, A. 2007, A\&A, 476, 1331

Press, W. H. 2002, Numerical Recipes (Cambridge: Cambridge Univ. Press), 785 Raassen, A. J. J., van der Hucht, K. A., Miller, N. A.,\& Cassinelli, J. P. 2008, A\&A, 478, 513

Rahoui, F., Chaty, S., Lagage, P., \& Pantin, E. 2008, A\&A, 484, 801

Rampy, R. A., Smith, D. M., \& Negueruela, I. 2009, ApJ, 707, 243 Rodriguez, J., Bodaghee, A., Kaaret, P., et al. 2006, MNRAS, 366, 274

Romano, P., Sidoli, L., Cusumano, G., et al. 2009a, MNRAS, 392, 45

Romano, P., Sidoli, L., Cusumano, G., et al. 2009b, MNRAS, 399, 2021

Romano, P., Sidoli, L., Krimm, H. A., et al. 2009c, Astr. Tel., 1961

Runacres, M. C., \& Owocki 2002, A\&A, 381, 1015

Sakano, M., Koyama, K., Murakami, H., Maeda, Y., \& Yamauchi, S. 2002, ApJS, 138,195

Sguera, V., Barlow, E. J., Bird, A. J., et al. 2005, A\&A, 444, 221

Sguera, V., Bazzano, A., Bird, A. J., et al. 2006, ApJ, 646, 452

Sguera, V., Hill, A. B., Bird, A. J., et al. 2007, A\&A, 467, 249

Sidoli, L., Romano, P., Mereghetti, S., et al. 2007, A\&A, 476, 1307

Sidoli, L., Romano, P., Mangano, V., et al. 2009a, ApJ, 690, 120

Sidoli, L., Romano, P., Ducci, L., et al. 2009b, MNRAS, 397, 1528

Smith, D. M., Main, D., Marshall, F., et al. 1998, ApJ, 501, 181

Smith, D. M., Heindl, W. A., Markwardt, C. B., et al. 2006, ApJ, 638, 974

Swank, J. H., Smith, D. M., \& Markwardt, C. B. 2007, Astr. Tel. 999

Tomsick, J. A., Chaty, S., Rodriguez, J., et al. 2009, ApJ, 694, 344

Vaughan, B. A., van der Klis, M., Wood, K. S., et al. 1994, ApJ, 435, 362

Zurita Heras, J. A., \& Chaty, S. 2009, A\&A, 493, L1

Zurita Heras, J. A., \& Walter, R. 2009, A\&A, 494, 1013

Walter, R., \& Zurita Heras, J. A. 2007, A\&A, 476, 335

Watanabe, S., Sako, M., Ishida, M., et al. 2006, ApJ, 651, 421

White, N. E., Swank, J. H., \& Holt, S. S. 1983, ApJ, 270, 711

White, N. E., Nagase, F., \& Parmar, A. N. 1995, X-ray binaries (Cambridge University, Press, ed. W. H. G. Lewin, J. van Paradijs, \& E. P. J. van den Heuvel, 1 